\title{
Fast and Slow Gamma Rhythms Are Intrinsically and Independently Generated in the Subiculum
}

\author{
Jesse Jackson, Romain Goutagny, and Sylvain Williams \\ Douglas Mental Health University Institute, McGill University, Department of Psychiatry, Montréal, Québec, Canada H4H 1R3
}

Gamma rhythms are essential for memory encoding and retrieval. Despite extensive study of these rhythms in the entorhinal cortex, dentate gyrus, CA3, and CA1, almost nothing is known regarding their generation and organization in the structure delivering the most prominent hippocampal output: the subiculum. Here we show using a complete rat hippocampal preparation in vitro that the subiculum intrinsically and independently generates spontaneous slow $(25-50 \mathrm{~Hz})$ and fast $(100-150 \mathrm{~Hz})$ gamma rhythms during the rising phase and peak of persistent subicular theta rhythms. These two gamma frequencies are phase modulated by theta rhythms without any form of afferent input from the entorhinal cortex or CA1. Subicular principal cells and interneurons phase lock to both fast and slow gamma, and single cells are independently phase modulated by each form of gamma rhythm, enabling selective participation in neural synchrony at both gamma frequencies at different times. Fast GABAergic inhibition is required for the generation of fast gamma, whereas slow gamma is generated by excitatory and inhibitory mechanisms. In addition, the transverse subicular axis exhibits gamma rhythm topography with faster gamma coupling arising in the distal subiculum region. The subiculum therefore possesses a unique intrinsic circuit organization that can autonomously regulate the timing and topography of hippocampal output synchronization. These results suggest the subiculum is a third spontaneous gamma generator in the hippocampal formation (in addition to CA3 and the entorhinal cortex), and these gamma rhythms likely play an active role in mediating the flow of information between the hippocampus and multiple cortical and subcortical brain regions.

\section{Introduction}

Both theta and gamma rhythms have been studied extensively in relation to memory processes in the rodent and primate brain (Jacobs et al., 2007; Montgomery and Buzsáki, 2007; Sederberg et al., 2007; Wyart and Tallon-Baudry, 2008; Jacobs and Kahana, 2009; Jutras et al., 2009; Tort et al., 2009; Rutishauser et al., 2010). Gamma rhythms in the hippocampal formation are important locally for cell assembly synchrony as well as globally for communication between disparate brain regions during various aspects of hippocampal-dependent memory (Montgomery and Buzsáki, 2007; Sederberg et al., 2007; Montgomery et al., 2008; Senior et al., 2008; Jutras et al., 2009; Tort et al., 2009). Increases in gamma power in cortical and hippocampal areas have been shown to improve spike timing accuracy and predict memory recall (Jutras et al., 2009). In the hippocampal formation, two main gamma bands are found in the slow (SG; 25-50 Hz) and fast (FG; 80-140 $\mathrm{Hz}$ ) frequency range, providing a frequency-specific transfer of information between hippocampal regions during different com-

\footnotetext{
Received March 16, 2011; revised June 9, 2011; accepted June 26, 2011.

Author contributions: J.J., R.G., and S.W. designed research; J.J. and R.G. performed research; J.J. contributed unpublished reagents/analytic tools; J.J. analyzed data; J.J. and S.W. wrote the paper.

This work was supported by the Canadian Institute of Health Research, the Natural Sciences and Engineering Research Council of Canada (NSERC), and the Fonds de la Recherche en Santé du Québec. R.G. was supported by the Conrad F. Harrington postdoctoral fellowship from the McGill Faculty of Medicine. J.J. received a Canadian Graduate Scholarship from NSERC. We thank Clayton Dickson, Guillaume Ducharme, Calvin Young, Amy Nakashima, and an anonymous reviewer for comments that greatly improved the clarity of this manuscript.

Correspondence should be addressed to Dr. Sylvain Williams, Douglas Mental Health University Institute, 6875 Lasalle Boulevard, Montréal, Québec, Canada, H4H 1R3. E-mail: sylvain.williams@douglas.mcgill.ca.

DOI:10.1523/JNEUROSCI.1370-11.2011

Copyright $\odot 2011$ the authors $\quad 0270-6474 / 11 / 3112104-14 \$ 15.00 / 0$
}

ponents of memory (Bragin et al., 1995; Sirota et al., 2008; Tort et al., 2008; Colgin et al., 2009). The SG band is thought to originate in CA3, whereas the medial entorhinal cortex (MEC) is likely involved in the generation of the FG rhythm (Bragin et al., 1995; Chrobak and Buzsáki, 1998; Colgin et al., 2009). These two gamma generators converge on CA1 circuits, providing slow and fast gamma modulation at different times (Colgin et al., 2009). Information contained within these generated gamma rhythms can then be conveyed to other cortical regions. However, between the hippocampus and the cortex resides the subiculum, which provides the primary and most divergent hippocampal output to entorhinal, parahippocampal, and diencephalic structures (Tamamaki and Nojyo, 1990; Witter and Groenewegen, 1990; Risold and Swanson, 1996; Naber and Witter, 1998; Naber et al., 2001; Kloosterman et al., 2003; Witter, 2006). Therefore, because CA1 and the MEC both converge on subicular circuitry, it would be expected that gamma activity of both slow and fast frequency would be present in the subiculum. However, investigation concerning the generation of gamma rhythms in the subiculum is sparse, particularly with regard to whether the structure spontaneously generates gamma oscillations in vitro or in vivo.

Using an isolated intact hippocampus in vitro, we measured the intrinsic capacity for theta and gamma rhythm generation within the hippocampal subregions of CA3, CA1, and the subiculum. Surprisingly, we found that network activity in the subiculum has a unique intrinsic structure facilitating robust gamma rhythm generation as well as theta-gamma coupling. This network organization plays an important role in mediating the strength and spatial coherence of spike timing precision of subic- 
ular circuits. Our findings provide evidence for a potentially important mechanism by which the coexpression of theta with two gamma frequency generators in the subiculum could facilitate hippocampal communication with a diversity of brain regions.

\section{Materials and Methods}

Dissection. All procedures were performed according to protocols and guidelines approved by the McGill University Animal Care Committee and the Canadian Council on Animal Care. Sprague Dawley rats (P15P28, male and female) were decapitated, and, after rapid removal from the skull, the brain was placed in an ice-cold, high-sucrose aCSF solution (in mM: 252 sucrose, $3 \mathrm{KCl}, 2 \mathrm{MgSO}_{4}, 24 \mathrm{NaHCO}_{3}, 1.25 \mathrm{NaH}_{2} \mathrm{PO}_{4}, 1.2$ $\mathrm{CaCl}_{2}$, and 10 glucose) that was bubbled with carbogen $\left(95 \% \mathrm{O}_{2}\right.$ and $5 \% \mathrm{CO}_{2}$ ). A detailed description of the dissection procedure can be found in recent reports (Khalilov et al., 1997; Wu et al., 2006; Goutagny et al., 2009). Note that, throughout Results and Discussion, the term hippocampus refers to the hippocampal proper including dentate gyrus, CA3, CA1, and subiculum, whereas the term hippocampal formation includes the parahippocampal and entorhinal cortex as well.

Data recording. During data recording, the preparation was continuously perfused with aCSF $(20-25 \mathrm{ml} / \mathrm{min} ; 126 \mathrm{~mm} \mathrm{NaCl}, 24 \mathrm{~mm}$ $\mathrm{NaHCO} 3,10 \mathrm{~mm}$ glucose, 3.5-4.5 mM KCl, $2 \mathrm{~mm} \mathrm{MgSO}_{4}, 1.25 \mathrm{~mm}$ $\mathrm{NaH}_{2} \mathrm{PO}_{4}$, and $2 \mathrm{~mm} \mathrm{CaCl}_{2}$, $\mathrm{pH} 7.4$, with $95 \% \mathrm{O}_{2} / 5 \% \mathrm{CO}_{2}$ ) via a gravityfed perfusion system and maintained at $30-32^{\circ} \mathrm{C}$. Local field potentials (LFPs) were recorded using glass micropipettes (1-4 M $\Omega$ ) filled with aCSF. Signals were recorded with a differential alternating current amplifier (A-M Systems), filtered online $(0.1-500 \mathrm{~Hz}$ ), and sampled at 5-10 $\mathrm{kHz}$. Signals were referenced to the bath medium and connected to ground. Local field potentials in the subiculum were recorded in the intermediate subiculum relative to the septal- temporal hippocampal axis and in the mid-subiculum relative to the transverse axis, unless indicated otherwise. Single units were recorded extracellularly using the same pipettes and acquired using online filters between $1 \mathrm{~Hz}$ and $10 \mathrm{kHz}$ and filtered offline between $0.3 \mathrm{kHz}$ and $10 \mathrm{kHz}$ to extract spike waveforms. Spiking activity was classified as single units only if there were no spikes in the 1-2 ms bins of the interspike interval (ISI) histogram. Putative interneurons and principal neurons were differentiated based in part on firing rate, spike width, and the presence of complex spike waveforms (Sharp and Green, 1994; Sharp, 1999; Brotons-Mas et al., 2010). Putative interneurons were taken to be only those cells that had a narrow spike width (peak-to-trough $<0.3 \mathrm{~ms}$ ) and a fast firing rate $(>20 \mathrm{~Hz})$. Extracellular principal cells were classified as those cells with broad action potential width $(\geq 0.7 \mathrm{~ms})$ and slow firing rate $(<7 \mathrm{~Hz})$. Bursting subicular neurons (non-interneurons with a short interspike interval in the 5-10 ms range) were typically excluded from the principal cell class because they had a higher firing rate of two to four spikes per burst.

Whole-cell recordings were performed using the blind patch technique as described previously (Manseau et al., 2008). Briefly, pipettes were pulled using borosilicate glass with a resistance of 3-8 $\mathrm{M} \Omega$ when filled with (in mM) $144 \mathrm{~K}$-gluconate, $3 \mathrm{MgCl}_{2}$, 0.2 EGTA, 10 HEPES, 2 ATP, and 0.3 GTP, pH 7.2 (285-295 mOsm). Recordings were performed using a patch-clamp amplifier (PC-505A; Warner Instruments). Cell characterization was performed by injecting $0-100$ pA, 2-s-long, depolarizing and hyperpolarizing current steps. Values reported were not corrected for the liquid junction potential of $13 \mathrm{mV}$.

Data analysis. For analyses involving only local field potentials, signals were down sampled to $1 \mathrm{kHz}$. Filtering in the specified frequency bands was performed in the forward and reverse direction to eliminate phase distortions, and the first and last second in the trace was removed. Power spectrums and spectral coherence were calculated using the Chronux signal processing toolbox (Bokil et al., 2010) using a time-frequency product of 3 and with five tapers. In the case in which time-frequency analysis was performed, we used a $4-8 \mathrm{~s}$ window and moved across the data in $1 \mathrm{~s}$ increments.

Gamma detection. We detected significant gamma events similar to previous studies (Csicsvari et al., 2003; Colgin et al., 2009). To detect gamma on each theta wave, we used the peak of theta as a reference and generated a spectrogram from -100 to $+100 \mathrm{~ms}$ relative the theta peak using a short-time FFT (with a $50 \mathrm{~ms}$ hamming window and $1 \mathrm{~ms}$ time step). The frequency with maximal power (between 20 and $250 \mathrm{~Hz}$ ) was recorded for each theta wave. For the calculation of the percentage of theta waves with SG, FG, or no gamma, we used the threshold technique (Csicsvari et al., 2003; Colgin et al., 2009). Gamma events in either the SG or FG frequency band were identified by taking the filtered $(25-55 \mathrm{~Hz}$ for SG and $80-180 \mathrm{~Hz}$ for FG) and rectified gamma LFP in the frequency range of interest, and events were detected as those crossing 2 SD above the mean of the rectified gamma signal. To avoid detecting multiple gamma events within one gamma burst, we required the gamma epoch to be separated by a time period proportional to the theta period (for example, $200 \mathrm{~ms}$ for a $5 \mathrm{~Hz}$ theta oscillation) calculated for each experiment based on the dominant theta frequency of the LFP autocorrelation. The relationship between $\mathrm{SG}, \mathrm{FG}$, and theta phase was assessed by calculating theta phase at the time point of maximal (above threshold) gamma amplitude during each theta cycle.

Gamma coherence was measured in three orientations relative to the subicular pyramidal cell layer. In a given experiment, coherence was measured in one or two of the axes, and the data were collapsed across experiments for analysis. Electrodes were moved in $150 \mu \mathrm{m}$ increments away from the reference electrode. In some cases, electrodes were instead moved toward the reference at the same interval spacing. All analyses were performed on the coherence values using the mean absolute distance and by detecting the peak coherence value in the theta, SG, or FG frequency bands.

Modulation index for cross-frequency coupling. The modulation index (MI) was calculated using the algorithm described previously (Tort et al., 2008). Similar to previous studies, we calculated the MI for pairs of frequencies, with amplitude frequencies $(f A)$ ranging from 5 to $250 \mathrm{~Hz}$ in $2.5 \mathrm{~Hz}$ increments and phase frequencies $(f p)$ ranging from 1 to $12 \mathrm{~Hz}$ in $0.5 \mathrm{~Hz}$ increments. The MI was calculated using the entropy measure $(H)$ taken from the following two equations:

$$
H=-\sum_{j=1}^{N} p_{j} \log p_{j}
$$

where $p_{j}$ is equal to

$$
p_{j}=\frac{<A_{f A}>\phi_{f p}(j)}{\sum_{j=1}^{N}<A_{f A}>\phi_{f p}(j)},
$$

and where $N$ is the number of phase bins $N=72$, which equates to using $5^{\circ}$ bins spanning from $-180^{\circ}$ to $180^{\circ}$.

$\left\langle\mathrm{A}_{f a}\right\rangle \phi_{f p}(j)$ denotes the mean amplitude value for frequency $\mathrm{A}_{f a}$ within the phase bin $j$ of phase frequency $\phi_{f p}(j)$.

The overall MI was calculated by expressing $H$ as a function of the maximum possible value, $H_{\max }$, the value obtained from a uniform distribution (where the amplitude values in all phase bins are equal), where $H_{\max }=\log N$. Therefore, MI $=\left(H_{\max }-H\right) / H_{\max }$. The MI is bound between 0 and 1 , where 0 would indicate equality to the uniform distribution (essentially the amplitude in all phase bins are equal). The greater the value of MI, the greater the distance from the normalized uniform distribution (for a graphical description of the method, see Tort et al., 2008).

The statistical significance of the MI was calculated by performing 200 surrogate MI calculations on the data, in which the gamma amplitude signal was shifted by randomly selected increments in both the forward and reverse direction, thereby generating a distribution of surrogate MIs. The obtained MI was required to be $>2$ SD above the mean surrogate MI to be considered statistically significant. For the theta-gamma MI analyses, the MI was calculated by using 3-5 min of continuous stationary data collected from activity generated after $1 \mathrm{~h}$ in the recording chamber. For the quantification of theta-gamma MI, the single filtered theta trace was used as the phase signal (with bandpass filters set -1 to $+1 \mathrm{~Hz}$ around the dominant theta frequency for that experiment), because we were principally interested in the MI at theta frequencies. The final MI 
value for SG and FG was taken as the integrated (summed) MI across the entire SG or FG frequency band.

The contribution of spikes to the MI measures has been shown to create "sharp edges" or other artifacts in the coupling index (Kramer et al., 2008; Colgin et al., 2009). We observed a similar phenomenon, which resulted from spectral leakage of the spike to all frequency bands and the inability to properly filter out such large-amplitude events with common bandpass filters. All experiments with such broadband coupling were removed, and traces were searched manually for filter performance, as well as for the presence of large-amplitude spikes. Data with sharp deflections arising from spikes were not used in the cross-frequency coupling analyses.

Isolated subiculum. Experiments in the isolated subiculum were conducted by removing the CA1-CA3 system at the time of dissection. To remove the subiculum, microscissors were moved through the border of CA1 and the subiculum while stabilizing the extracted hippocampus with forceps. Preparations were allowed to recover for $\geq 1 \mathrm{~h}$ before being placed in the recording chamber and for an additional 30-60 min before recordings were performed. For the comparison of gamma amplitude and theta-gamma coupling, intact hippocampal isolates from the other hemisphere of the same animal or a littermate were used.

Single-cell analysis. For extracellular spiking analysis, 3-8 min of continuous data were used for each unit, depending on the firing rate. The cells were located predominately within the pyramidal layer of the midsubiculum in the region of subicular tissue in which the largest gamma amplitude was present (the first 200-400 $\mu \mathrm{m}$ from subicular surface). For the visualization of spike phase locking to theta, SG, and FG spikes were binned into 12 bins of $30^{\circ}$ each. The phase vector of each voltage time series was computed using the Hilbert transform in Matlab (MathWorks) for each network rhythm (theta, SG, and FG). The Circstat toolbox was used for circular statistics, including circular mean, mean resultant length, and circular correlation coefficient between phase angles (Berens, 2009). For all plots, $0^{\circ}$ indicates the oscillation peak.

To analyze the relationship between instantaneous gamma amplitude and spike phase locking, the SG or FG amplitude and phase at each spike time were collected, and phase locking for the highest 33\% of gamma amplitude events (strong gamma) and lowest 33\% gamma amplitude events (weak gamma) were compared with the average resultant length for the individual cell. Therefore, the same number of spikes was used for the calculation of spike phase locking for both strong and weak gamma.

To determine the dependence of phase locking in one gamma frequency on the amplitude in the other frequency band, three independent spike-gamma conditions were used: (1) SG only, (2) FG only, and (3) SG together with FG. A spike was classified as arising during one of the above conditions if the instantaneous gamma amplitude at the time of spiking was $>1.5 \mathrm{SD}$ from the mean rectified gamma trace. Here, the threshold was lowered from 2 SD to include more spikes in all conditions. This type of analysis can assess whether phase locking in one gamma frequency band can arise independent of activity in the other gamma band. For example, in the SG-only condition, phase locking to SG was assessed by only using spike times when the SG amplitude was above threshold and the FG amplitude was below threshold. Likewise, the FG-only condition included spikes that occurred during above-threshold FG and belowthreshold SG. This detection procedure inevitably yields a different number of spikes for each gamma condition, and the total number of spikes influenced the mean resultant length. Therefore, the number of spikes for all conditions was equalized by limiting the number of spikes, $n$, in each condition by the lowest spike count of the three conditions. In the remaining categories, $n$ spikes were selected randomly from the total spike count, and the resultant length was calculated using this dataset. This randomization procedure was repeated 100 times for each dataset, and the mean resultant length from this 100 point matrix was taken as the "grand mean" for the specific gamma category of that experiment. The overall resultant length for every cell (ignoring gamma amplitude) was also calculated using the random selection of $n$ spikes (as above) from the entire dataset and calculating the resultant length on this subset, to keep the number of spikes used in all analyses constant.

Depth profile analysis. Depth profiles through the subiculum were performed using a 16-channel silicon probe with $100 \mu \mathrm{m}$ spacing
(Neuronexus Technologies) that was lowered perpendicular to the hippocampal surface. The transmembrane current flow was calculated using the formula $\operatorname{CSD}=\operatorname{lfp}\left(p_{i}\right)-2 \times \operatorname{lfp}\left(p_{i-2}\right)+\operatorname{lfp}\left(p_{i+1}\right) / d^{2}$, where CSD is current source density, $\operatorname{lfp}\left(p_{i}\right)$ is the field potential recorded at probe channel $i$, and $d$ is the distance between channels (in this case, $100 \mu \mathrm{m}$ ). The CSD of theta, SG, and FG were calculated using the filtered gamma traces in the respective frequency band, triggered by a reference electrode located within $100 \mu \mathrm{m}$. The presence of significant currents was detected at each depth by taking the absolute value of the filtered gamma LFP (in either SG or FG) using the Hilbert transform (Matlab). Significant gamma currents at each depth were detected by requiring that the CSD at given depth was above a threshold equal to the mean \pm 3 SD. This analysis assessed the spatial spread of gamma current generation across the deep-superficial axis.

Histology. After the completion of an experiment, the hippocampi were fixed in $4 \%$ paraformaldehyde followed by sucrose until saturated. Tissue was cut $(50 \mu \mathrm{m})$ at $-20^{\circ} \mathrm{C}$ with a cryostat to visualize the electrode placement. The tissue was cut in either the coronal or horizontal orientation, mounted on gelatin-coated slides, and stained with either cresyl violet or fast red.

Statistical analysis. Data were collected from a total of 101 single isolated hippocampal explants, and data from all of these cases were included in the basic description of subicular gamma. Subsets of hippocampi were used for other various experiments and are presented throughout. For the single-unit data, only one cell was recorded per electrode track. All data were analyzed using Matlab using custom-made programs and scripts published previously (Tort et al., 2008; Berens, 2009; Tort et al., 2009). Paired $t$ tests were used to evaluate differences between conditions, and the corresponding $p$ values were adjusted using the Bonferroni's correction for multiple comparisons. Data are presented as mean \pm SE unless otherwise indicated.

\section{Results}

\section{Spontaneous fast and slow gamma in the subiculum}

Using the isolated rat whole-hippocampal preparation (see Methods and Materials) (Khalilov et al., 2003; Wu et al., 2006; Goutagny et al., 2009), we recorded local field potentials throughout the hippocampus (CA3, CA1, and subiculum) because spontaneous theta activity is present in this in vitro model (Goutagny et al., 2009). Our aim was to determine whether gamma frequency oscillations co-occurred with theta rhythm in the intact isolated hippocampus. The amplitude of both SG $(25-55 \mathrm{~Hz})$ and FG $(80-180 \mathrm{~Hz})$ was simultaneously measured in CA3, CA1, and the subiculum $(n=11)$ during persistent theta rhythms $(3-10 \mathrm{~Hz})$ (Fig. 1). All recordings were performed approximately halfway between the septal and temporal hippocampal poles, and, in the subiculum, recordings were made in the mid-subiculum (with reference to the transverse axis). It was found that there was significantly greater gamma amplitude in the subiculum (mean amplitude, $30.4 \pm 0.7 \mu \mathrm{V}$ for SG and $27.9 \pm 0.4 \mu \mathrm{V}$ for FG) relative to that in $\mathrm{CA} 1\left(t_{(10)}=4.43, p=0.0016\right.$ for SG; $t_{(10)}=$ $4.68, p=0.0011$ for FG) and CA3 $\left(t_{(10)}=4.1, p=0.0028\right.$ and $t_{(10)}=$ 2.64, $p=0.0268$ for SG and FG, respectively) (Fig. $1 B$ ). Gamma rhythms were coexpressed with theta rhythm in the subiculum in $>90 \%$ of preparations and persisted for $30-120 \mathrm{~min}$.

\section{Phase amplitude cross-frequency coupling in the subiculum}

Theta-gamma coupling is thought to have an especially important function in mediating hippocampal-cortical (Sirota et al., 2008; Tort et al., 2008; Colgin et al., 2009) and hippocampalsubcortical (Tort et al., 2008) communication during learning (Tort et al., 2009), episodic memory (Lisman and Idiart, 1995; Senior et al., 2008), working memory (Sauseng et al., 2009), and recall tasks (Canolty et al., 2006; Tort et al., 2009). We were therefore interested in determining whether the cross-frequency coupling between theta phase and gamma amplitude was expressed intrinsically in the subiculum or in the CA1 or CA3 subfields as 
A

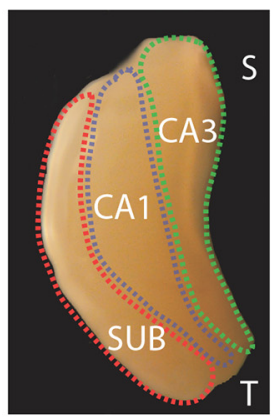

B

SG amplitude FG amplitude

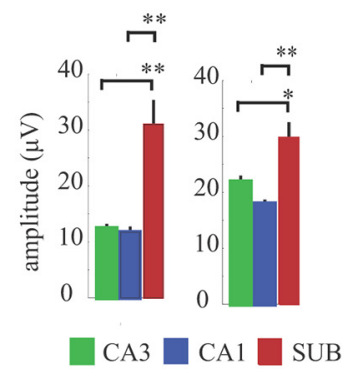

C

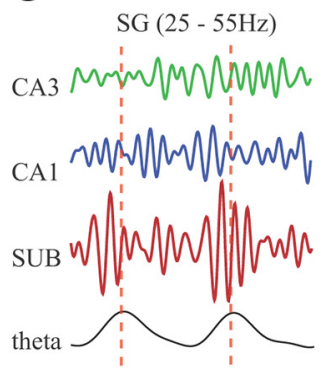

D
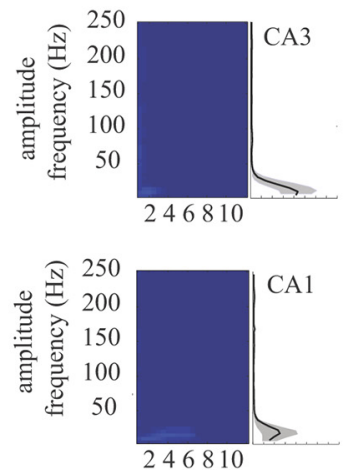

FG $(80-180 \mathrm{~Hz})$
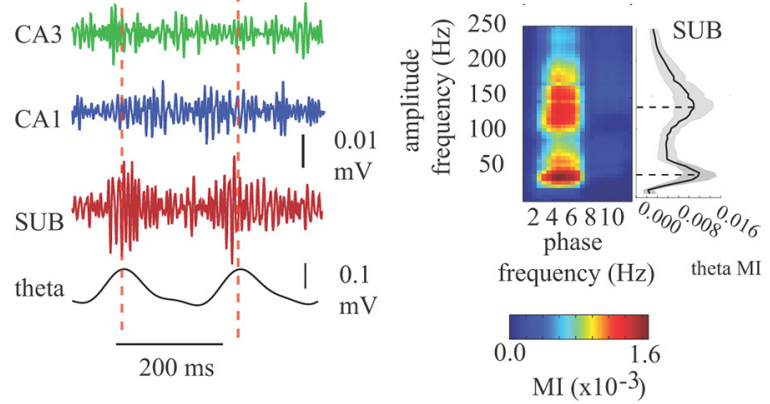

E

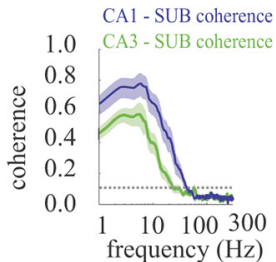

F

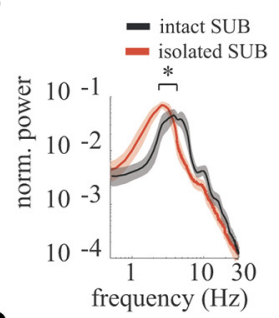

G

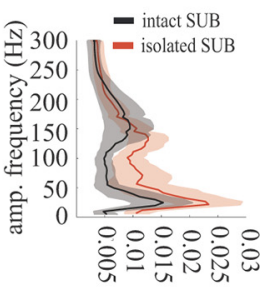

theta MI

Figure 1. Intrinsic fast and slow gamma coupling is a unique property of subicular circuits. A, Diagrammatic representation of the isolated hippocampus and the locations in which LFPs were simultaneously measured from the subiculum (SUB), CA1, and CA3 and the septal (S) and temporal (T) end of the hippocampus. $\boldsymbol{B}$, Mean \pm SE amplitude of gamma bursts detected in the three regions. $C$, Example filtered LFP data simultaneously recorded from the SUB, CA1, and CA3 in the SG, FG, and theta bands. The filtered theta trace was taken from the subiculum. The red dashed lines are shown to visually align the traces to the peak of theta. $\boldsymbol{D}$, Example cross-frequency coupling plots from CA3 (top), CA1 (middle), and SUB (bottom). Adjacent to the plots is the mean \pm SE theta phase modulation index for 11 experiments for each region. $\boldsymbol{E}$, Significant theta coherence was present between the subiculum and $C A 3$ and subiculum and $C A 1$, and theta coherence was greater between $\mathrm{CA} 1$ and subiculum. However, frequencies above $30 \mathrm{~Hz}$ were not coherent between the subiculum and $\mathrm{CA} 1$ or CA3, suggesting that gamma does not arise from the CA3-CA1 axis. $F$, Mean power spectrums from experiments in which the subiculum was isolated compared with the intact hippocampus. Data for these experiments were collected from hippocampi in which the subiculum was removed from CA1 and compared with data collected from the hippocampi of the opposite hemisphere or from a littermate on the same day of recording. Theta frequency was reduced when the subiculum was isolated, but intrinsic theta was still present. $\mathbf{G}$, The mean \pm SE theta phase MI plots for experiments with the isolated subiculum and intact preparation. Theta phase- gamma amplitude coupling was still present in the isolated subiculum. ${ }^{*} p<0.05,{ }^{* *} p<0.01$.

well. To test this and to quantify the degree with which the two gamma frequencies were coupled to theta phase, the MI technique (Tort et al., 2008, 2009) was used to measure the magnitude of phase locking between the amplitude of activity in the 5-250 $\mathrm{Hz}$ frequency band (in $2.5 \mathrm{~Hz}$ steps) to the phase of lowfrequency LFP activity (ranging from 1 to $12 \mathrm{~Hz}$ in $0.5 \mathrm{~Hz}$ steps). We found that the subiculum generated cross-frequency coupling plots resembling that reported in rat CA1 (Sirota et al., 2008; Tort et al., 2008; Colgin et al., 2009) and human neocortical recordings (Canolty et al., 2006), with distinct bimodal peaks in the MI distribution at $32 \pm 2$ and $130 \pm 2 \mathrm{~Hz}(n=101, p<0.05)$ (see Methods and Materials) (Fig. 1D). However, the presence of this coordinated form of activity was not consistently observed in the CA1 or CA3 area (Fig. 1D), suggesting that a unique form of intrinsic processing can take place in the subiculum, at the final output stages of the hippocampus. The FG theta phase MI was significantly higher in the subiculum than in CA3 or CA1 $\left(t_{(10)}=\right.$ $3.89, p<0.01$ and $\left.t_{(10)}=3.90, p<0.01\right)$, whereas the SG theta phase $\mathrm{MI}$ in the subiculum was higher than in CA3 $\left(t_{(10)}=2.55\right.$, $p<0.05$; the CA1 - subiculum SG MI difference was not significantly different, $p>0.05$ ).

\section{Cross-frequency theta-gamma coupling in the subiculum is intrinsic}

Although there was significant LFP coherence in the theta band between the subiculum and CA1 $(0.84 \pm 0.06)$ and the subiculum and CA3 $(0.68 \pm 0.04)$, coherence above $20-30 \mathrm{~Hz}$ dropped below the significance threshold, suggesting that inputs from the CA1-CA3 system were not responsible for subicular gamma (Fig. $1 E$ ). To completely rule out that gamma and theta-gamma coupling was not attributable to afferent inputs from the CA3-CA1 system, the subiculum was isolated during the dissection procedure (see Methods and Materials). The peak theta frequency was reduced in the preparations without the CA3-CA1 complex $\left(-1.3 \mathrm{~Hz}, t_{(10)}=3.27, p<0.01\right)$ (Fig. $\left.1 F\right)$. However, the mean amplitude of SG and FG ( $p>0.05$ for both SG and FG) and the strength of phase-amplitude coupling were unchanged when the CA3-CA1 system was removed ( $p>0.05$ for both SG and FG) (Fig. $1 G$ ). Therefore, the subiculum can intrinsically generate spontaneous SG and FG without input from the CA1 or MEC (because the MEC is removed in the dissection procedure in all preparations in this study), suggesting that a unique network architecture exists within the subiculum that spontaneously expresses these dynamic activity patterns.

\section{Covariation in SG and FG spectral power}

We also measured how both SG and FG covaried in terms of spectral power. If SG and FG were generated by the same underlying mechanisms then they should undergo similar changes in power across time. Measurements were made regarding how all frequencies (from 1 to $250 \mathrm{~Hz}$ ) covaried in terms of spectral power, using the correlation coefficient method described previ- 

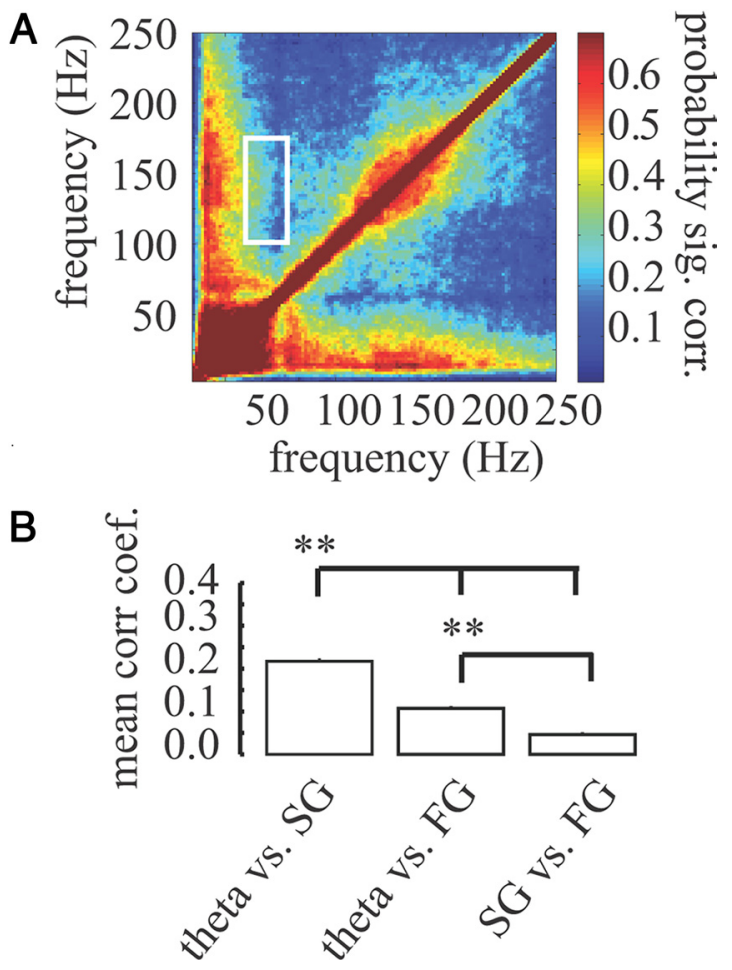

Figure 2. Slow and fast gamma rhythm power fluctuate independently. $\boldsymbol{A}$, The crosscorrelation matrix expressing the probability of significant amplitude-amplitude coupling between all frequencies (from 1 to $250 \mathrm{~Hz}$ ) from all experiments $(n=101)$. This matrix represents the probability that the spectral amplitude of each pair of frequencies would be significantly correlated in an experiment. The matrix is symmetrical across the diagonal. Clusters near the diagonal reflect neural rhythms and clusters off the diagonal reflect covariation in power between two frequencies (for example theta and FG). The white box outlines the region highlighting the correlation between SG power and FG power, indicating that there is a very low covariation in power and therefore these two frequencies undergo independent dynamic changes in amplitude. $\boldsymbol{B}$, The mean $\pm \mathrm{SE}$ correlation coefficients for the correlation between theta and SG (left), theta and FG (middle), and SG and FG (right). ${ }^{* *} p<0.001$.

ously (Masimore et al., 2004). Frequencies around SG and FG tended to form local clusters on the comodulogram (Fig. 2A), therefore indicating the presence of network rhythms in the respective frequency bands. Furthermore, theta power covaried with both SG (mean, $r=0.21 \pm 0.02)$ and FG $(r=0.10 \pm 0.01)$, because clusters also arose at the intersection of theta frequencies and both SG and FG. However, the dynamics of SG and FG were only weakly correlated (mean, $r=0.065 \pm 0.01$ ), demonstrating that fluctuations in SG and FG are independent (Fig. $2 B$ ).

\section{Depth profile of subicular rhythms}

CSD analysis was performed using a 16-channel silicon probe that was lowered through the deep-superficial axis of the subiculum during ongoing theta and gamma rhythms $(n=5)$ (Fig. $3 A, B)$. Theta, $\mathrm{SG}$, and FG were all generated within the same region between the pyramidal layer and deep molecular layer. However, the maximal sink/source alternation for theta and SG was located predominantly in the molecular layer, whereas the maximal FG CSD was located closer to the pyramidal cell layer (Fig. 3C). Therefore, SG and FG differed slightly in the spatial extent of gamma currents. To measure how SG and FG current amplitudes were related, significantly large SG and FG current bursts were detected at each depth. The threshold for significance was defined as current amplitudes exceeding 3 SD above the mean absolute current for each channel. We measured the FG current amplitude during SG current bursts and SG current amplitude during significant FG bursts. If SG and FG arise from the same mechanism, then it would be expected that the large FG bursts would accompany SG bursts (and vice versa). There was no consistent correlation between SG and FG during either significant SG current bursts (Fig. 3D, left) or during significant FG current bursts (Fig. 3D, right). Therefore, in the isolated hippocampus during persistent theta rhythm (Goutagny et al., 2009), the subiculum is the most robust gamma generator producing gamma in both SG and FG frequency bands. These data show that, in addition to areas CA3 (Fisahn et al., 1998; Csicsvari et al., 2003; Oren et al., 2006; Montgomery and Buzsáki, 2007; Colgin et al., 2009) and the MEC (Bragin et al., 1995; Chrobak and Buzsáki, 1998; Cunningham et al., 2003, 2004; Colgin et al., 2009; Quilichini et al., 2010), the subiculum is a third generator of gamma in the rodent hippocampal formation and that it generates gamma in two distinct frequency bands.

\section{Independent fast and slow theta-gamma coupling}

It was next determined how the frequency of theta differentially impacted SG and FG coupling dynamics. Experiments were grouped according to their preferred theta frequency (Fig. $4 A-$ $C)$. Faster theta frequencies $(\geq 7 \mathrm{~Hz}$ ) were accompanied by faster peak frequency of SG modulation $(40 \pm 3 \mathrm{~Hz})$ compared with slower theta $\left(\leq 4.5 \mathrm{~Hz}, 26 \pm 2 \mathrm{~Hz}, t_{(51)}=4.3, p<0.001\right)$ or mid-range theta frequencies $(\leq 7 \mathrm{~Hz}$ and $4.5 \mathrm{~Hz}, 31 \pm 2 \mathrm{~Hz}$, $\left.t_{(69)}=4.1 p<0.001\right)$. In contrast, the frequency of FG theta phase modulation was unrelated to theta frequency. In addition, the magnitude of the SG MI was greater for slower theta than faster theta $\left(t_{(51)}=3.8, p<0.001\right)$ (Fig. $4 C$ ), and the magnitude (or depth) of the FG MI did not change between theta frequencies. These results suggest that the cross-frequency, theta gamma coupling regimen changes according to theta frequency, with lower theta frequencies facilitating coupling at SG frequencies whereas with faster theta frequencies, FG is the more dominant coupling frequency (Fig. $4 C$ ). Therefore, faster theta frequencies are accompanied by a greater ratio of FG to SG. To further examine the relationship between SG MI and FG MI, we performed multiple linear regression to analyze the predictors of SG MI or FG MI dynamics across time (Fig. 4D). The best predictor of SG MI or FG MI was gamma power in the respective frequency band. Additionally, the gamma MI in one frequency band did not assist in the prediction of the MI in the other (again supporting SG-FG independence). Finally, to examine the possibility that FG may just be a harmonic of SG, we determined the distance (in hertz) of the FG peak frequency from the predicted SG harmonics. In only $10 \%$ of all cases did the FG peak frequency coincide with a harmonic of SG (usually the third or fourth harmonic), but on average, the FG frequency was $7.7 \pm 0.6 \mathrm{~Hz}$ from the closest SG harmonic ( $p<0.001$; significantly different than 0$)$ (Fig. $4 E$ ). Therefore, on average, FG is not a harmonic of SG.

The phase amplitude comodulation plots in Figure 4 suggest that the two forms of gamma in the subiculum are independent. To definitively assess the number of theta cycles with SG, FG, or both, we used a time window proportional to the theta period (see Methods and Materials) (Colgin et al., 2009) and calculated the time-frequency spectral power $(20-250 \mathrm{~Hz})$ across each theta cycle. Within each theta cycle, the significance of SG and or FG amplitude was determined by calculating the maximal gamma amplitude for each theta cycle and then determining whether the amplitude was above the significance threshold (2 SD above the mean). The gamma detection window was limited to a period proportional to the theta cycle to avoid detecting multiple peaks 


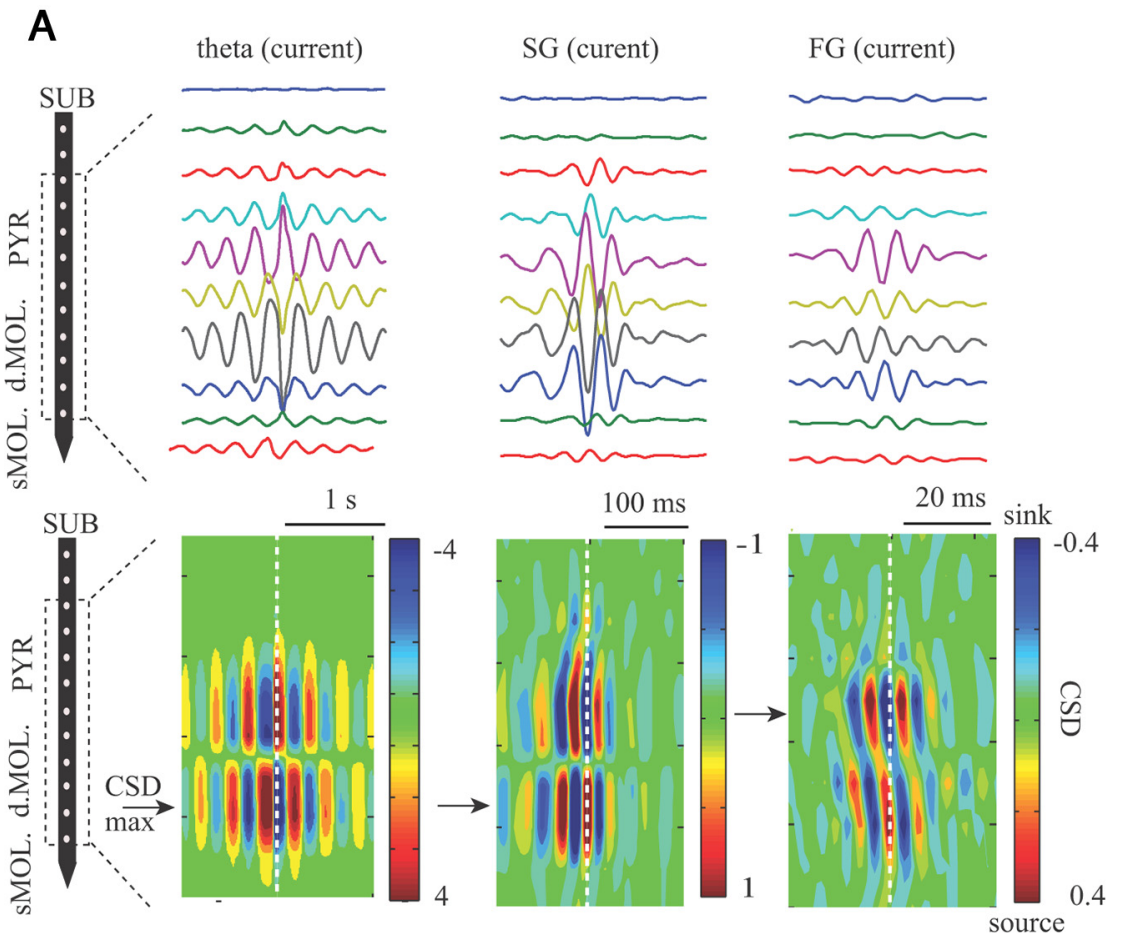

B

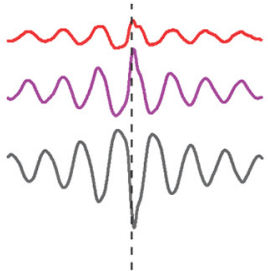

C theta (CSD) $\quad$ SG (CSD) FG (CSD)
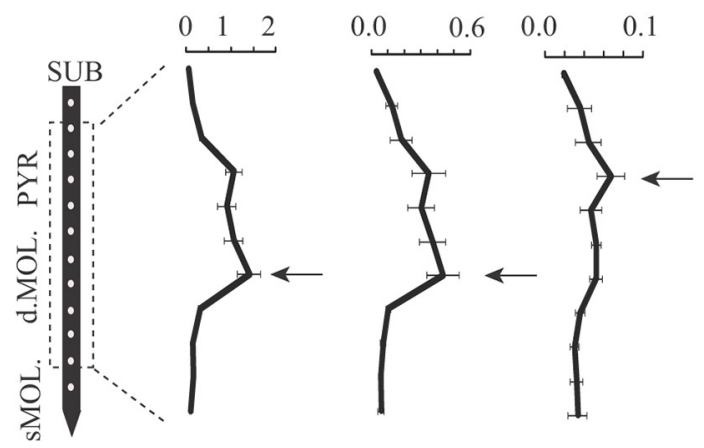

D $\mathrm{SG} \rightarrow \mathrm{FG} \mathrm{FG} \rightarrow \mathrm{SG}$

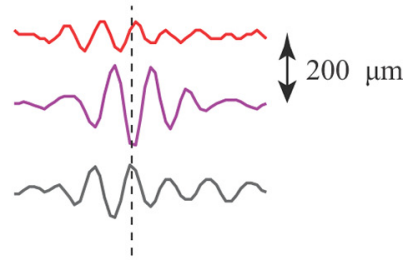
corr. coef. (r) corr. coef. (r)

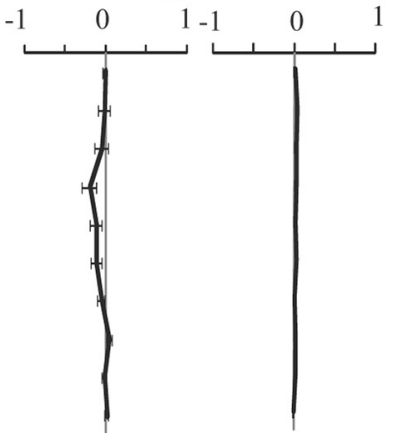

Figure 3. Current source density of subicular rhythms. A, CSD was performed through the deep-superficial axis of the subiculum(SUB), using a reference electrode located just below the pyramidal cell layer (PYR). Shown are representative CSD sor one experiment for theta, SG, and FG. The mean current traces are shown above the heat maps for the electrode locations indicated on the left. A reference electrode located near the 16-channel probe was used to trigger the data from $3 \mathrm{~min}$ of a continuous recording. To generate the averages, the peak of the filtered theta, $\mathrm{SG}$, or FG was detected in the reference, and the CSD in all channels was collected centered on these events (white dashed lines). The black arrows indicate the maximum (SD alternation for each frequency band. $\boldsymbol{B}, A$ closer look at the data in $\boldsymbol{A}$, showing the presence of phase shifts for all three rhythms. The dashed line indicates the time of the peak theta, SG, or FG LFP recorded from the reference electrode and used to trigger the current from each channel for the three frequencies. $C$, The mean absolute CSD fluctuations across depth. Note that currents for all rhythms are maximally located within the same $500 \mu \mathrm{m}$, but the FG current maximum is closer to the pyramidal cell layer. $D$, The correlation coefficient between $S G$ and $F G$ current magnitude using SG current peaks (left) and FG current peaks (right). SG and FG current peaks were detected using an amplitude threshold (mean \pm 3 SD). This analysis therefore measures the covariation between $\mathrm{SG}$ and $\mathrm{FG}$ current during large-amplitude current bursts of each frequency.

within the same gamma burst. Theta cycles with different timefrequency signatures were present (Fig. 5A), but, most importantly, theta cycles with SG did not necessarily have FG (and vice versa). Both SG and FG were coupled to a similar phase of theta rhythm, although FG tended to occur slightly later within the theta cycle (Fig. $5 B)\left(-50 \pm 2^{\circ}\right.$ for $\mathrm{SG}$ vs $-43 \pm 2^{\circ}$ for $\mathrm{FG}$, and $F_{(1,100)}=7.8, p=0.006$, WatsonWilliams test for two samples of angles) (Berens, 2009). Averaged across all experiments, the percentage of theta cycles with SG alone, FG alone, SG and FG together, and no gamma was $12.2 \pm 0.3,36.1 \pm 1.3$, $31.3 \pm 1.4$, and $20.4 \pm 1.2 \%$, respectively. Therefore, the number of theta cycles with independently occurring SG or FG was greater than the number of theta cycles in which SG and FG concomitantly occurred (Fig. $5 C)\left(t_{(100)}=9.87, p<0.001\right)$. Because both forms of gamma arise on similar phases of the theta cycle, it was determined whether the amplitude of SG and FG covaried during those theta waves in which both SG and FG were present. As expected based on the data in Figure $5 D$, there was no significant correlation between SG and FG peak amplitude (mean, $r=0.04 \pm 0.01, p>0.05, n=101$ ) (Fig. 5D). SG and FG can arise on the same theta cycle in the subiculum, unlike in CA1 in which SG and FG occur mostly on different theta cycles and in which SG and FG power are negatively correlated (Colgin et al., 2009). Furthermore, the two forms of gamma reported here are independent, because FG is not required for SG generation, $S G$ is not required for FG generation, and, in instances in which they occur together, the SG and FG amplitude are not related. Therefore, although on average these two forms of gamma both occur on the rising phase and peak of theta rhythms, they do so at slightly different phases and with unrelated amplitudes.

\section{Phase locking of principal cells and interneurons to fast and slow gamma} It was next determined how principal cells and interneurons fired in relation to SG and FG phase. To do so, we analyzed the spiking activity of putative principal cells $(n=17)$ and interneurons $(n=5)$ recorded within the pyramidal layer of subiculum (Fig. 6) (see Methods and Materials for cell selection procedure). Fastspiking interneurons fired with a peak first-order ISI at 7-9 ms, corresponding to the FG frequency range (Fig. $6 \mathrm{~B}$ ). Principal cells had a peak ISI in the SG range (20-40 ms). Of the principal cells identified, 14 of 17 phase locked to SG and 11 of 17 exhibited significant phase locking to FG. Although there was substantial variability in the preferred phase of principal spiking for SG and FG (Fig. $6 C$ ), on average principal cells fired with the greatest phase modulation near the peak of SG $\left(66 \pm 33^{\circ}\right)$ and FG $\left(116 \pm 34^{\circ}\right)$ (Fig. 6C,E). Fast-spiking interneurons, however, fired near the 
A
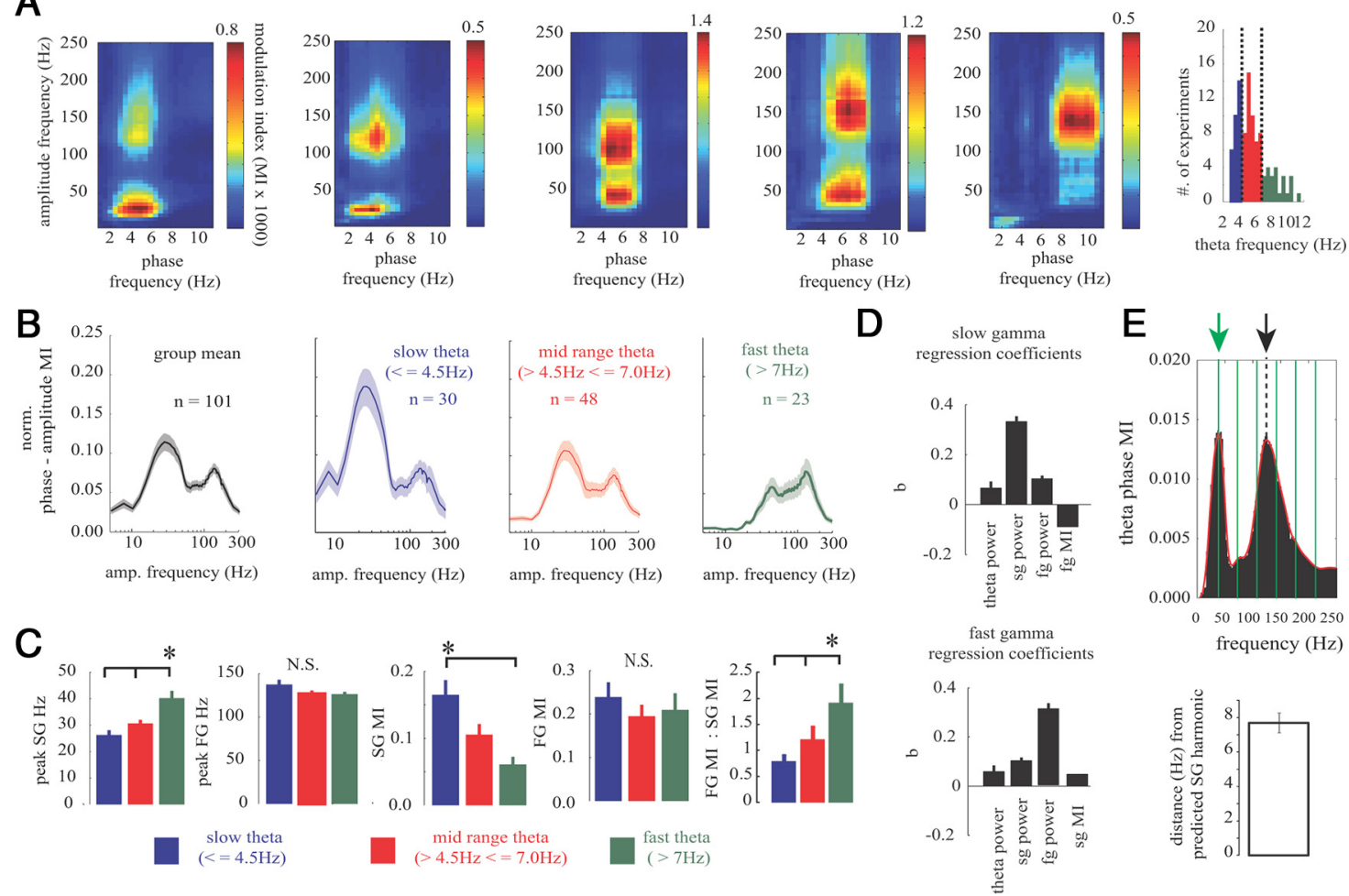

Figure 4. Slow and fast gamma independently phase lock to theta rhythms. A, Example cross-frequency coupling plots showing the amplitude modulation of all frequencies (5-250 $\mathrm{Hz}$ ) by the phase of low frequencies (1-12 Hz). Five examples are shown to highlight the effect theta frequency has on the gamma coupling dynamics. The plots are ordered from slower to faster theta frequency (left to right). Note that slower theta frequencies had greater SG coupling, whereas fast theta frequencies were dominated more by FG. The color map depicts the strength of phase-amplitude coupling between pairs of frequencies calculated using the modulation index (see Methods and Materials). The scale is the MI $\times 1000$. On the right is the histogram of preferred theta frequency in all experiments $(n=101)$. Theta frequency was divided into three frequency bands for the comparison of theta- gamma modulation index analyses. The vertical lines indicate the cutoff frequencies used for analysis. $\boldsymbol{B}$, The theta phase modulation index (spanning $5-300 \mathrm{~Hz}$ ) for the three theta frequency bands. Note the change in SG modulation across theta frequency. Dark lines are the mean across the number of experiments indicated, and shaded regions are the SE. C, Quantification of the peak SG and FG frequency across the different theta frequencies. The peak SG frequency increased as theta frequency increased. Right, The magnitude of phase modulation decreased with greater theta frequency for SG but not FG. The ratio of FG MI to SG Ml increased with increased theta frequency. $\boldsymbol{D}$, The factors predicting changes in SG and FG amplitude modulation to theta phase. The mean \pm SE $\beta$ coefficients (b) from multiple linear regression analysis using SG MI (top) or FG MI (bottom) as the dependent variable and theta power, SG power, FG power, and SG MI (top) or FG MI (bottom) as predictors. To obtain the data for each regression analysis, the power and MI was calculated in $6 \mathrm{~s}$ bins over the duration of $5-30$ min recordings. The best predictor of $S G M I$ was $S G$ power, suggesting that increases in $S G$ power were associated with a stronger phase coupling to theta phase. Note that the FG MI did not help in the prediction of SG MI. The FG MI was also best predicted by FG power rather than theta power, SG power, or SG MI. $E$, FG does not arise from a harmonic of $S G$. Shown is an example plot of the theta phase $\mathrm{Ml}$ across frequencies from 5 to $250 \mathrm{~Hz}$. The green arrow is the dominant $S G$ frequency, and the solid green lines are the five first harmonics of the main SG frequency. If FG arises from a harmonic of SG, the FG peak will align with one of the SG harmonics, which is not the case here (black arrow). Below is the mean \pm SE minimum difference between the $\mathrm{FG}$ peak frequency and closest SG harmonic. ${ }^{*} p<0.001$.

trough and falling phase of SG $\left(-174 \pm 21^{\circ}\right)$ and FG $\left(122 \pm 22^{\circ}\right)$ (Fig. 6D,E). Therefore, the interaction between principal cells and interneurons is likely occurring during the ongoing rhythms in both SG and FG frequencies.

We confirmed that both excitatory and inhibitory events were responsible for slow and fast gamma rhythms by performing whole-cell recordings from principal cells in the subiculum during the spontaneously occurring theta and gamma activity $(n=$ 14 cells) (Fig. $6 F-I$ ). Cells had a resting membrane potential of $-49 \pm 1.5 \mathrm{mV}$ (range, -47 to $-61 \mathrm{mV}$, not corrected for the liquid junction potential) and could fire spontaneously in 11 of 14 cases. We only analyzed cells in which the resting membrane potential demonstrated a significant degree of theta coherence with the extracellular field ( 7 of 14 cells) (Fig. 6F, G). We examined the synaptic activity in subicular principal cells at -70 and 0 $\mathrm{mV}$ during extracellularly recorded theta-gamma activity. These two membrane potentials were chosen to principally record EPSPs at $-70 \mathrm{mV}$ (the null potential of $\mathrm{GABA}_{\mathrm{A}}$-mediated IPSPs under our recording conditions) and IPSPs at $0 \mathrm{mV}$ (the reversal potential of EPSPs). At $-70 \mathrm{mV}$, postsynaptic potentials in both the SG and FG frequency range were phase locked to theta $(p<$
0.05, Rayleigh's test for significance) (Fig. $6 H$ ), similar to the extracellular potentials. Similarly, inhibitory synaptic events were also found to be organized in the SG and FG range $(p<0.05)$ (Fig. $6 \mathrm{H}$ ), again confirming that both EPSPs and IPSPs were phase locked to theta, similar to the extracellular LFPs. The dynamics of EPSPs and IPSPs within each theta cycle were next examined relative to the LFP data as performed in Figure 5. Single theta cycles were more likely to have either SG or FG synaptic potentials than both $(p<0.05)$ (Fig. $6 I)$.

\section{Subicular neurons independently participate in both slow and fast gamma synchrony}

In CA 1 , it was shown recently that single neurons prefer to phase lock to one form of gamma rhythm (fast or slow) while avoiding phase entrainment by the other (Colgin et al., 2009). Because the subiculum is positioned downstream to CAl, it is set to receive both SG and FG forms of activity. Therefore, it may be that subicular neurons phase lock to only SG or FG. As discussed above, both interneurons and pyramidal cells phase locked to SG and FG. Principal cells phase locked more robustly to SG than FG $\left(n=10, t_{(9)}=2.5, p=0.03\right.$, including only cells that phase 
A
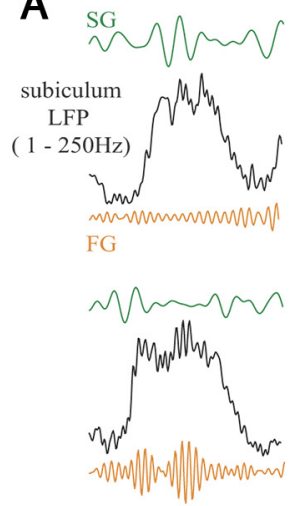

whm

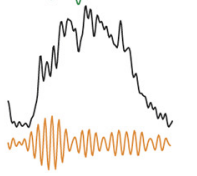

nmon

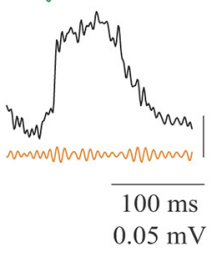

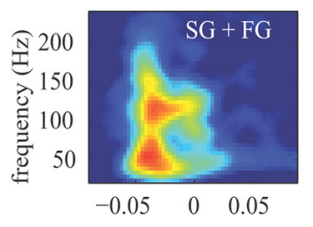

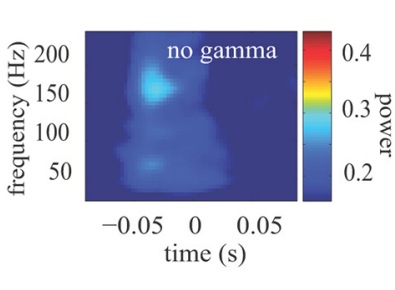

B
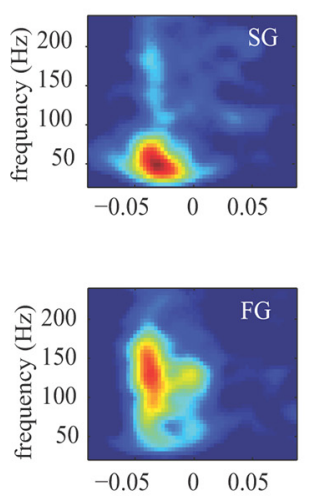

D
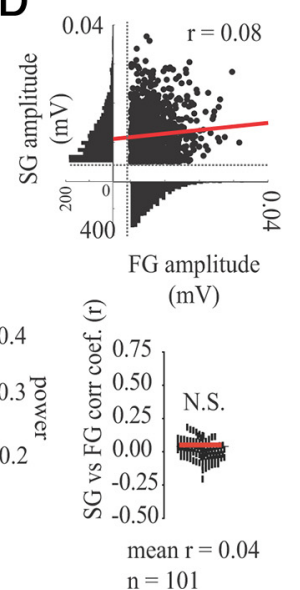

Figure 5. Slow and fast gamma independently arise within the theta cycle. $\boldsymbol{A}$, Four theta cycles are shown (top to bottom) with significant SG only, FG only, both SG and FG, or no gamma. On the right, the mean average short time Fourier transform generated spectrogram centered on theta peak (time 0 ) for all waves during one experimental session. Mean spectrograms were created from all theta waves with SG only, FG only, both SG and FG, or no gamma. $\boldsymbol{B}$, Distribution of the preferred theta phase for SG or FG for all experiments. FG occurred slightly later in the theta cycle. $C$, The percentage of theta cycles with either $S G$ or $F G$ was greater than the number of theta cycles with both forms of gamma. $D$, The amplitude of co-occurring SG ( $y$-axis) and FG ( $x$-axis) is shown for one experiment for each theta cycle with both forms of gamma, and a correlation coefficient was calculated between the maximum amplitudes of SG and FG. The red line is the linear fit. Below is the scatter plot of the correlation coefficient between SG and FG amplitude for all experiments showing that the peak amplitudes of SG and FG were not related. The red line indicates the grand experimental mean. ${ }^{*} p<0.01$.

locked to both), whereas the phase-locking strength between SG and FG for interneurons was not different $(n=5, p>0.05)$. Furthermore, there was no difference in SG or FG phase locking between principal cells and interneurons ( $p>0.05$ for both). We analyzed spike-gamma phase-locking dynamics (including all cell types) and found that 40 of 49 neurons phase locked to SG, FG, or both. Of these phase-locked neurons, 36 of 40 phase locked to SG, 33 of 40 phase locked to FG, and a large degree of overlap existed between the two populations (29 of 40). Therefore, in contrast to CA1, the same subicular neurons participate in synchrony at both gamma frequencies. It would be important for SG and FG within subiculum to be independent, because the frequency of CA1 input (SG or FG) may indicate different memory states (retrieval or encoding, respectively) (Colgin et al., 2009). Therefore, we analyzed the independence of phase locking between SG and FG for all neurons that phase locked to both rhythms (Fig. 7A) $(n=29)$. The SG phase and FG phase at the time of spiking was not related $(n=29$; mean circular, $r=0.05$, $p>0.05$ ), indicating that knowledge of the phase of firing within the $\mathrm{SG}$ or FG cycle did not assist in determining the phase of firing in the other frequency band. This is likely because the peak amplitude (as shown previously; Figs. 2, 5) of SG and FG within the

theta cycle did not covary. It was next determined whether the amplitude of SG and FG predicted the strength of gamma phase locking. Previous reports have shown that increases in gamma amplitude facilitate spike- gamma phase locking (Tukker et al., 2007; Oren et al., 2010). Corroborating these findings, it was found that stronger SG or FG increased the spike phase locking to the respective frequency band (Fig. $7 B$ ). For example, spiking during a strong (large-amplitude) SG burst of activity was more phase locked to SG than during smalleramplitude SG events $\left(t_{(28)}=13.3, p<\right.$ $0.001)$. The same was true for FG $\left(t_{(28)}=\right.$ $6.8, p<0.001)$. Because $S G$ and FG power are primarily independent, these data suggest strong phase locking in one frequency may arise without phase locking in the other. To conclusively test whether spikegamma phase locking was independent between frequency bands, epochs were identified in which spiking occurred during isolated SG (with weak FG), isolated FG (with weak SG), or during both SG and FG (Fig. 7C). The number of spikes and spike phase locking was computed for each of these conditions and compared with the overall average phase locking of the particular cell (using the mean resultant length). The number of spikes that occurred during both SG and FG was reduced relative to isolated FG and isolated $S G\left(t_{(28)}=6.27, t_{(28)}=5.12, p<0.001\right.$ for each) (Fig. 7D). Therefore, although cells can phase lock to both rhythms, they typically only participate in one gamma frequency within a single theta cycle. Importantly, all cells exhibited significant phase locking to SG during reduced FG, demonstrating that SG phase locking can arise without FG. Similarly, cells maintained their phase locking to FG during reduced amplitude SG. Interestingly, the co-occurrence of SG and FG increased SG phase locking relative to SG alone (Fig. $7 E)\left(t_{(28)}=\right.$ $4.29, p<0.001$ ), demonstrating a facilitation of SG phase locking by the presence of FG. Although FG facilitates spike-SG phase locking, this facilitation would occur in a limited number of instances because more spikes arise during isolated SG than during the co-occurrence of SG and FG (Fig. 7D). In contrast, there was no increase in FG phase locking during concomitant SG-FG epochs. These data demonstrate that independent spike phase synchrony can occur in one gamma band without activity in the other frequency band. Furthermore, FG facilitates SG phase locking, but the reverse is not true. Therefore, the same subicular neuron(s) can independently participate in network synchrony at SG, FG, or both at different times, allowing the dynamic participation in both network rhythms.

\section{Differential role for excitation and inhibition in slow and fast gamma}

Various forms of hippocampal gamma rhythms have been identified in different in vitro preparations (Whittington et al., 1995; 
A

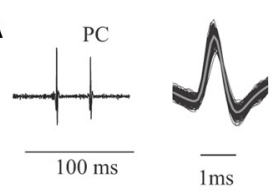

B

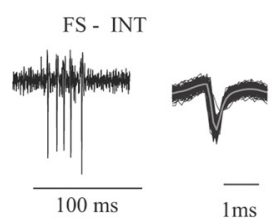

$\mathrm{F}$
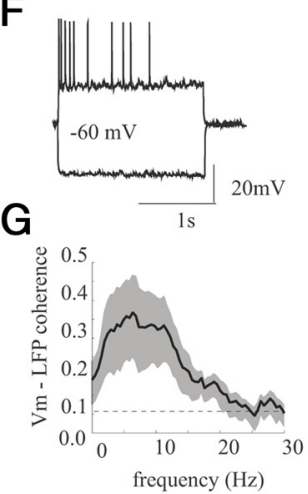

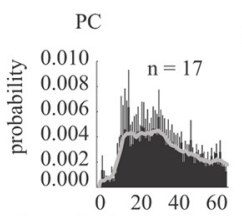

interspike interval (ms)

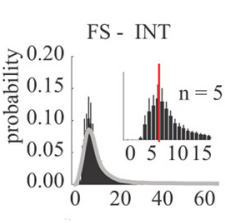

interspike interval (ms)
C

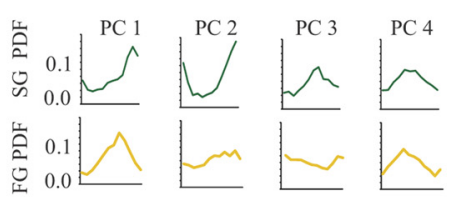

D

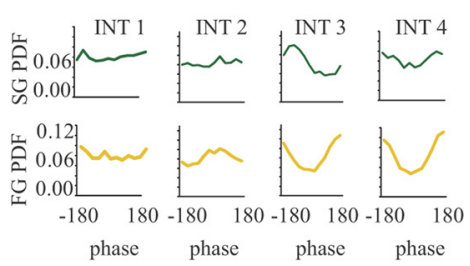

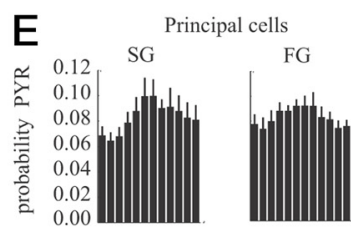

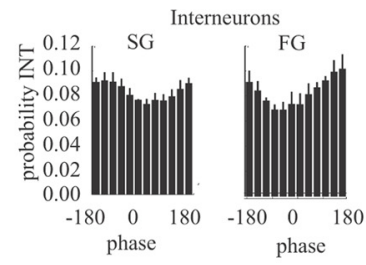

H slow gamma

fast gamma EPSPs EPSPs

slow gamma fast gamma

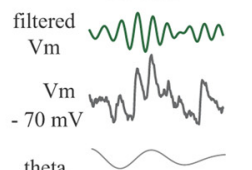

lfp
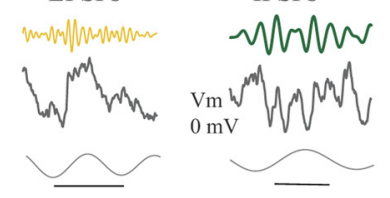
IPSPS
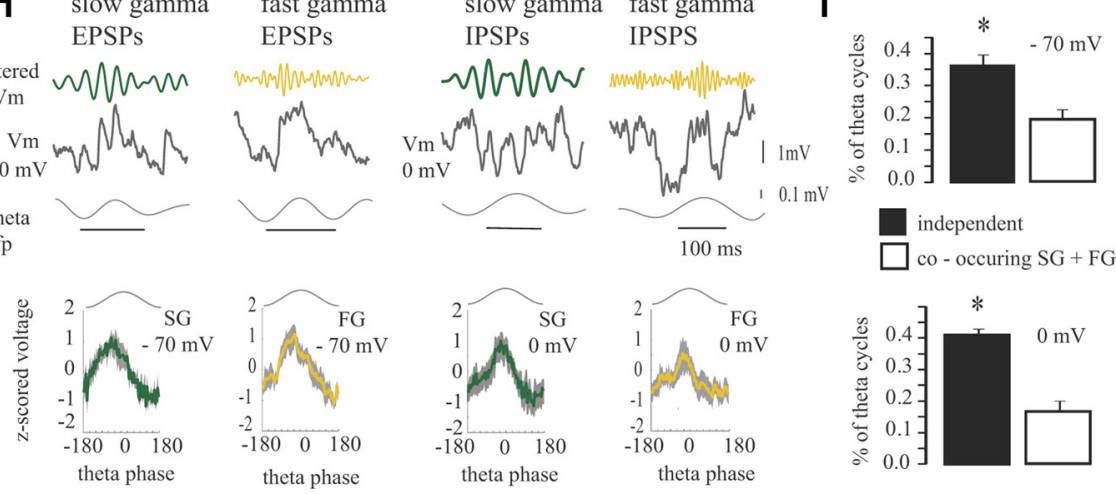

Figure 6. Phase locking of excitatory and inhibitory events to slow and fast gamma. Raw data and mean spike waveform for a representative principal cell (PR, $A$ ) and fast-spiking interneuron (INT, B) On the right is the mean ISI for all principal cells (top) and interneurons (bottom). Note the peak in the interneuron ISI at FG periods (expanded in the inset), whereas the principal cells have a broad distribution over the $20-40 \mathrm{~ms}$ range. $\boldsymbol{C}, \boldsymbol{D}$, The spike phase probability distribution for eight subicular neurons, including four principal cells (PC, $\boldsymbol{C}$ ) and four interneurons (INT, $\boldsymbol{D})$ for slow and fast gamma. $E$, Mean \pm SE phase-locking histograms for all principal cells and interneurons during SG and FG. Note that, although the preferred phase is somewhat diverse, principal cells on average fire near the peak of $S \mathrm{G}$ and $\mathrm{FG}$, whereas on average, interneurons show the greatest depth of modulation on the trough of both $\mathrm{SG}$ and $\mathrm{FG}$. $\boldsymbol{F}$, An example whole-cell recording of a principal cell in the subiculum during depolarizing and hyperpolarizing current steps. $\boldsymbol{G}$, Mean $\pm \mathrm{SE}$ coherence between the whole-cell recording and extracellular LFP. $\boldsymbol{H}$, Postsynaptic potentials recorded at both $-70 \mathrm{mV}$ (left) and $0 \mathrm{mV}$ (right). Example theta cycles are shown, demonstrating that both SG and FG potentials occur during the rising phase of the theta rhythm, similar to extracellular events. Traces above the raw $V_{\mathrm{m}}$ were bandpass filtered in the SG (green) and FG (gold) frequency bands. Below is the mean \pm SE of the normalized voltage fluctuations within the theta cycle for $\mathrm{SG}$ and $\mathrm{FG}$ at both 0 and $-70 \mathrm{mV}$. The plot shows the mean $z$-scored voltage for the envelope of the filtered $\mathrm{SG}$ or $\mathrm{FG}$ signal. $I$, The percentage of theta cycles with statistically significant $S \mathrm{G}$ or $\mathrm{FG}$ is greater than the number of cycles with both forms of gamma at both holding potentials. ${ }^{*} p<0.05$.

Traub et al., 2004; Oren et al., 2006). Depending on the region and experimental conditions, gamma oscillations of various frequencies from 20 to $80 \mathrm{~Hz}$ have been identified. One model of gamma generation involves feedback between principal cells (excitatory cells) and inhibitory interneurons (Fisahn et al., 1998; Traub et al., 2004; Tiesinga and Sejnowski, 2009). Another popular gamma model arises from the tonic depolarization of inhibitory interneurons that can maintain gamma rhythms in the absence of fast glutamatergic receptor activation (Whittington et al., 1995). We tested the effect of bath application of DNQX (10 $\mu \mathrm{M}$, to block AMPA/kainate receptors) and bicuculline (BMI) ( $10 \mu \mathrm{M}$, to block $\mathrm{GABA}_{\mathrm{A}}$ receptors) on gamma power. Both drugs penetrate the hippocampal tissue rapidly, within $1 \mathrm{~min}$ of bath application (Goutagny et al., 2009, their supplemental Fig. 9). DNQX reduced the power of $S G\left(t_{(7)}=3.4, p<0.05\right)$ but failed to significantly reduce FG power (Fig. 8). BMI reduced both SG $\left(t_{(5)}=3.1, p<0.05\right)$ and FG $\left(t_{(5)}=3.77, p<0.01\right)$ power (Fig. 8). Neither DNQX nor BMI eliminated all network activity in either SG or FG frequency bands, although when the two drugs were applied simultaneously, all activity was abolished (Fig. 8). The NMDA receptor antagonist D-APV $(50 \mu \mathrm{M})$ had no effect on SG or FG power $(p>0.05)$. Therefore, although both principal cells and interneurons participate in SG and FG during spontaneous conditions, FG can be generated without fast glutamatergic synaptic transmission.
Topographical organization of gamma rhythms in the subiculum

Anatomical studies have shown the existence of a columnar-like organization among subicular principal cells and between principal cells and interneurons (Harris and Stewart, 2001; Harris et al., 2001; Menendez de la Prida, 2003). These separate modules are thought to exist in both the proximal-distal and septal-temporal subicular axes and maintain non-overlapping parallel pathways connecting neocortical and subcortical areas serving different brain functions (Witter et al., 1990; Naber et al., 2001; Kloosterman et al., 2003). Therefore, we investigated whether gamma rhythms could provide an electrophysiological correlate for the anatomical modules previously reported. We measured the degree of neural communication (using spectral coherence) in three dimensions of subicular space spanning the proximaldistal ( $n=7$ preparations), septal-temporal $(n=9$ preparations), and deep-superficial ( $n=9$ preparations) axes. The LFP coherence was measured at theta, SG, and FG frequencies between a reference electrode, and electrodes were placed sequentially in $150 \mu \mathrm{m}$ increments in these three axes (Fig. 9A,B). Theta coherence was high $(>0.9)$ at all distances measured (Fig. 9B). SG maintained significant coherence values up to $750 \mu \mathrm{m}$ from the reference electrode in the proximal-distal direction and septaltemporal direction, whereas FG coherence fell below the significance threshold after $300 \mu \mathrm{m}$ on average, suggesting that, unlike 
A

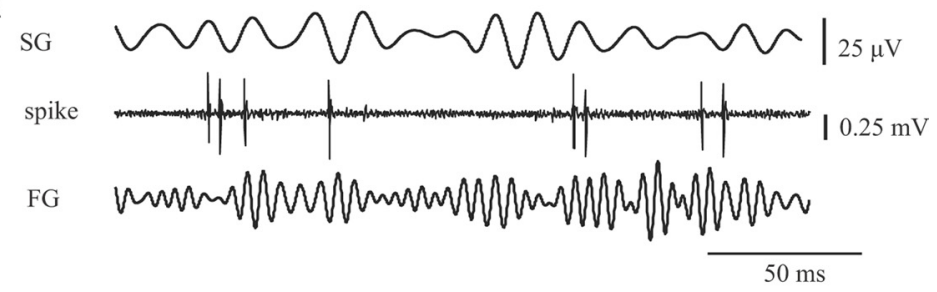

B
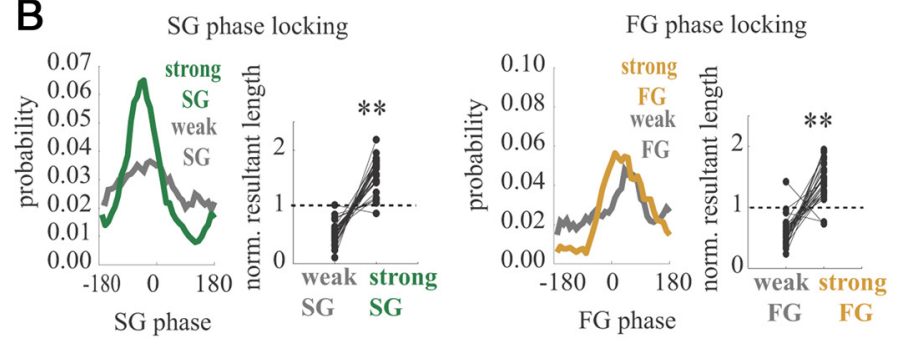

C

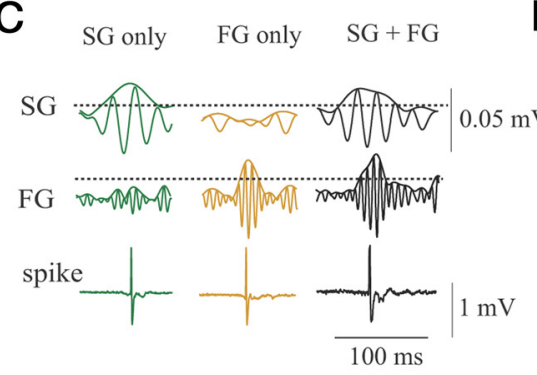

D $\quad *$

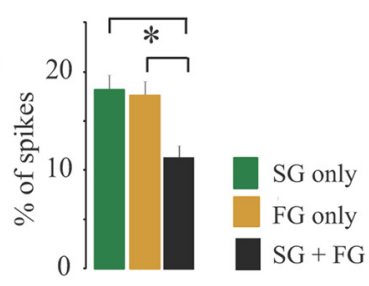

$\mathrm{E}$
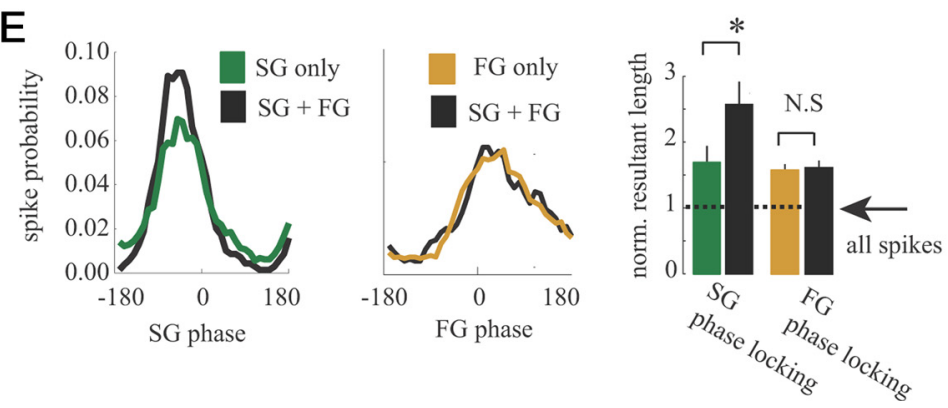

Figure 7. Slow and fast gamma independently modulate spike phase locking in the same neural network. $\boldsymbol{A}$, Top, An example trace showing spiking from a subicular principal cell and the simultaneously recorded SG and FG from a nearby ( $0.1 \mathrm{~mm})$ electrode. $\boldsymbol{B}$, Left, Spikes arising during stronger SG were more tightly phase locked to SG phase. The same trend was found for FG (right). Spike phase locking was compared during "strong" and "weak" amplitude activity in the respective frequency band using the same number of spikes (see Materials and Methods). The overall mean phase-locking value for each cell was normalized to 1. Note that almost all cells are above the mean value for strong gamma and below the mean for weak gamma. $\boldsymbol{C}$, Shown are three representative data epochs showing the simultaneously recorded slow gamma (SG, top), fast gamma (FG, middle), and principal cell spiking (bottom, recorded with a separate pipette). The gamma traces shown were bandpass filtered in the SG (25-55 Hz) or FG (80-180 $\mathrm{Hz}$ ) frequency range. The $\mathrm{SG}$ and $\mathrm{FG}$ amplitude envelope is also shown on top of each filtered gamma trace and was calculated using the absolute value of the Hilbert transformed signal. The three traces represent the three conditions used for analyzing spikephase locking during the presence or absence of either SG or FG (not shown is the presence of spiking during no gamma). The presence or absence of gamma in each band at the time of each spike was determined by an amplitude threshold (see Materials and Methods). $\boldsymbol{D}$, The number of spikes recorded during each of the different gamma conditions. Spikes were more likely to fire during SG or FG in isolation then during co-occurring SG and FG. $E$, Phase locking of spikes to SG (left) or FG (middle) during the presence or absence of each form of gamma rhythm. On the left is the phase-locking probability for one neuron for SG and FG during the states indicated. Note the increase in SG phase locking during spikes occurring with both SG and FG, whereas FG phase locking does not exhibit this facilitation. Right, Group data across all neurons. Similar to above, the overall mean resultant length was normalized to 1; therefore, a value of 1 would indicate that the strength of phase locking did not change above the overall experimental mean resultant length (using all spikes regardless of gamma amplitude), and values 1 would indicate that phase locking in the specific condition was increased. ${ }^{*} p<0.01,{ }^{* *} p<0.001$.

theta and SG, FG is best suited to organize small discrete regions of the subiculum (Naber et al., 2001; Kloosterman et al., 2003). There was a significant main effect of axis direction for SG $\left(f_{(2,110)}\right.$ $=29.5, p<0.001)$ and FG $\left(f_{(2,110)}=42.6, p<0.001\right.$ ) (for follow-up $t$ test values, see Fig. 9B), demonstrating that the gamma coherence in both frequencies did not change uniformly in all directions and that gamma coherence was mainly organized across the pyramidal cell layer in the deepsuperficial axis. Both SG coherence and FG coherence were most robust in the deep-superficial axis relative to the septaltemporal $\left(t_{(16)}=3.79, p<0.01\right.$ for SG, $t_{(16)}=4.7, p<0.001$ for FG) and proximal-distal axis $\left(t_{(14)}=2.8, p<0.05\right.$ for SG; $t_{(14)}=3.8, p<0.01$ for FG). The SG coherence observed at greater spatial locations could be attributable to volume conduction of the larger SG signal (relative to $\mathrm{FG}$ ), suggesting that the difference in SG and FG phase synchronization measured between regions is not necessarily physiologically meaningful. We therefore measured spike phase locking (using the mean resultant length) in a subset of neurons (including all cell types) between the local LFP and a more distant electrode recording $(1 \mathrm{~mm}$, located in either the septaltemporal or proximal-distal axes) (Fig. $9 C)$. The majority of neurons $(62 \%)$ exhibited significant SG phase locking to the distant subicular region, although the strength of SG phase locking was reduced $\left(t_{(20)}=5.0, p<0.001\right.$, comparing the mean resultant length for $S G$ between the local and distal LFP electrode). However, only $14 \%$ of the cells were significantly phase locked to FG in the distant subicular region, and the phase locking was reduced in all but one cell $\left(t_{(20)}=4.99 p<0.001\right.$; relative to the local LFP FG recording). Therefore, in addition to LFP coherence (Fig. 9B), more neurons were synchronized to SG in the distant subicular regions than to FG in distant regions $(p<$ 0.01 , binomial test), demonstrating that SG exerts a spatially greater synchronizing influence than FG (Fig. 9C). Therefore, the slab or columnar organization of subicular modules (Tamamaki and Nojyo, 1990) could be supported physiologically by FG but less so by SG.

The topography of neural inputs and outputs in the proximal-distal subicular axis exhibit a precise anatomical organization (Naber et al., 2001; Kloosterman et al., 2003). We found that the proximaldistal subiculum could be dissected with regards to the preferred frequency of its gamma modulation (Fig. 9D,E). The proximal subiculum (closer to CA1) exhibited a stronger ratio of SG to FG theta phase coupling strength relative to the distal subiculum $\left(t_{(11)}=\right.$ $3.29, p<0.007$ ) (Fig. 9D), demonstrating a proximal-distal organization in the preferred frequency of hippocampal output along transverse axis. Furthermore, the distal subiculum had a 
higher FG frequency $(135 \pm 9 \mathrm{~Hz})$ than the proximal subiculum $\left(114 \pm 8 \mathrm{~Hz}\right.$; $t_{(11)}$ $=2.3, p<0.05$ ), whereas the SG frequency did not differ in the proximal-distal axis (Fig. 9E). Theta frequency was not different between the proximal and distal subiculum $(p>0.05)$. This frequency difference at FG supports the lack of FG coherence proximo-distally and suggests that independent FG generators allows these regions to perform local processing without interference from highfrequency activity in the adjacent subicular module. This topographical organization of gamma frequencies could play an important role in differentially communicating with postsynaptic regions such as the lateral and medial entorhinal cortex, which are known to receive inputs from the proximal and distal subiculum, respectively (Kloosterman et al., 2003). To summarize, subicular gamma rhythms are geometrically organized in spatially defined modules with synchronization arising most robustly in the deep-superficial axis perpendicular to the pyramidal cell layer and with a topographical organization of gamma frequencies that correspond to functionally different subicular regions.

\section{Discussion}

We have demonstrated here that the hippocampal outputs from the subiculum spontaneously generate two main frequencies of gamma rhythms in the intact hippocampus in vitro similar to the gamma frequencies reported in vivo (Bragin et al., 1995; Sirota et al., 2008; Tort et al., 2008; Colgin et al., 2009; Sullivan et al., 2011). Interestingly, we also show the first evidence of spontaneous theta-nested slow and fast gamma oscillations in vitro. These gamma rhythms were present without any form of pharmacological or electrical stimulation, suggesting that this is the true default mode of the subicular network. The subiculum is the main hippocampal output, and therefore hippocampal-cortical and hippocampal-subcortical communication arises most predominantly from subicular neurons. These results support the idea that a significant amount of information processing in the subiculum arises independent of CAl or MEC inputs (Deadwyler and Hampson, 2004; Cappaert et al., 2007), suggesting an important role for subicular local circuits in mediating hippocampal outputs. These gamma rhythm-generating circuits likely have a role in mediating hippocampal-dependent behaviors, such as spatial memory, emotion, stress, fear, and reward (Herman and Cullinan, 1997; Witter, 2006; O’Mara et al., 2009; van Strien et al., 2009).

\section{The subiculum is a gamma generator}

Slow and fast gamma band frequency rhythms have been reported in the hippocampal formation. It is now well established that the CA3 area contains an SG band generator (Bragin et al., 1995; Oren et al., 2006; Colgin et al., 2009). This SG generator can be activated in the CA3 area of hippocampal slices with bath
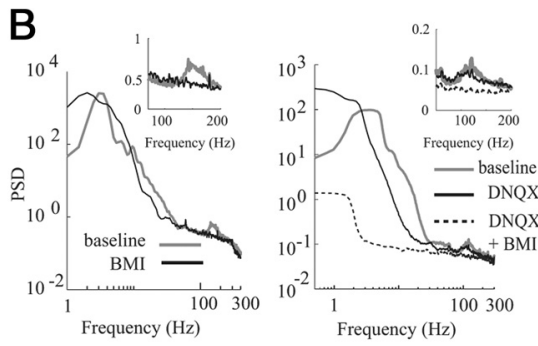

\section{C}
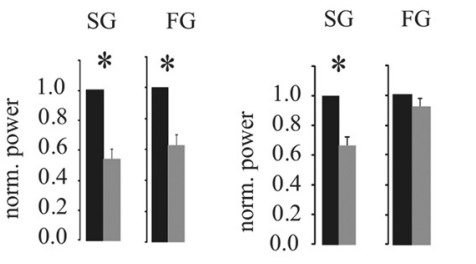

baseline BMI

Daseline $\square$ DNQX
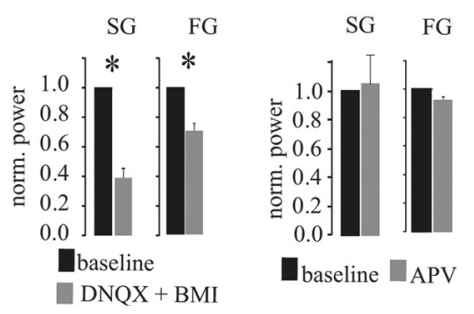

DNQX + BM
0.8

0.4

0.2

0.0

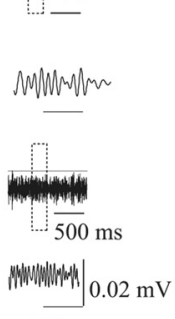

$100 \mathrm{~ms}$

Figure 8. Excitation and inhibition differentially control slow and fast gamma. A, Pharmacology of $S G$ and FG. Field potentials were analyzed during baseline and during the application of drugs to block fast GABAergic (BMI, $10 \mu \mathrm{M}$ ) and glutamatergic (DNQX Thansmission. Filtered traces within each frequency band and conditions are shown. The red lines indicate the threshold power was reduced by DNQX (top right). Coapplication of BMI and DNQX reduced SG and FG power (bottom left). Neither SG nor FG was on average reduced by NMDA receptor blockade using APV (bottom right). ${ }^{*} p<0.05$.

application of carbachol or kainate and requires balanced excitation and inhibition between principal cells and interneurons (Atallah and Scanziani, 2009). Similarly, the SG spontaneously generated here within the subiculum was also mediated through the activation of AMPA and $\mathrm{GABA}_{\mathrm{A}}$ receptors. Others have previously reported slow gamma frequency activity in the subiculum after tetanic stimulation of the CA1 area (Colling et al., 1998; Funahashi and Stewart, 1998; Stanford et al., 1998). The gamma frequency events in these reports appear to be different from the SG reported here because they are very-large-amplitude transient population events often characterized by spike doublets and are never observed to be nested with theta oscillations. However, these population gamma events share a similar pharmacological profile to the SG here (i.e., sensitive to both AMPA and GABA receptor antagonists). Conversely, the hippocampal FG $(>80$ $\mathrm{Hz}$ ) is thought to arise extrinsically in the MEC (Bragin et al., 1995; Chrobak and Buzsáki, 1998; Colgin et al., 2009). To our knowledge, this is the first report of spontaneous, intrinsically generated FG frequency band oscillations in the hippocampus. Fast-spiking interneurons all phase locked to FG, and FG power and local synchrony were reduced by $\mathrm{GABA}_{\mathrm{A}}$ receptor blockade, suggesting that rapid and strong local inhibition in the subiculum (Menendez de la Prida, 2003) is primarily responsible for FG activity. Therefore, it appears that the two forms of gamma in 
A

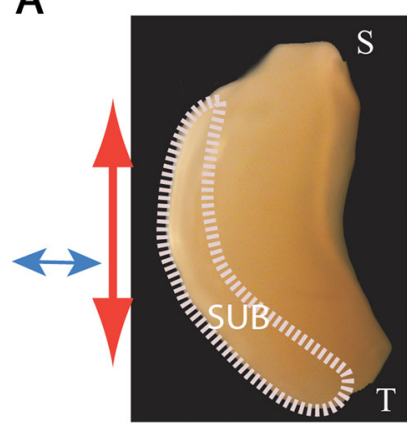

B

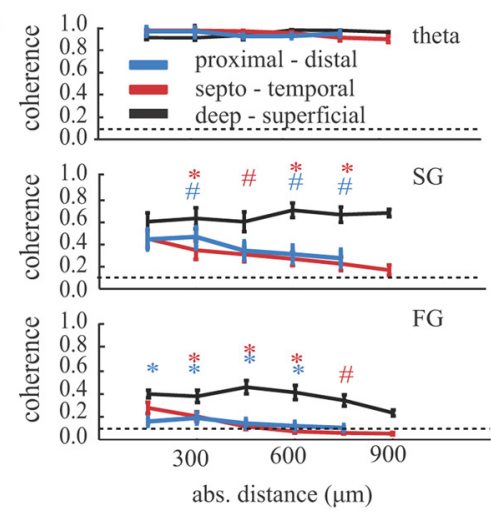

C

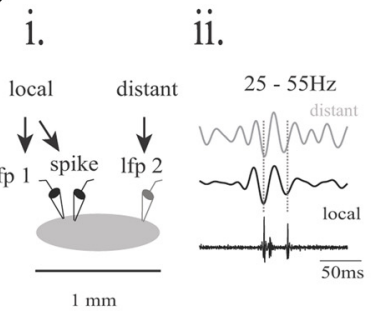

ii.

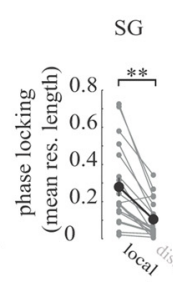

iii.

111.

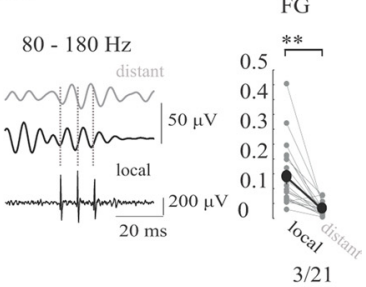

$\mathrm{D}$
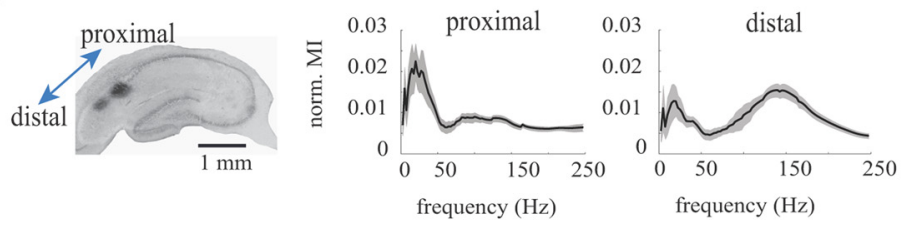

E
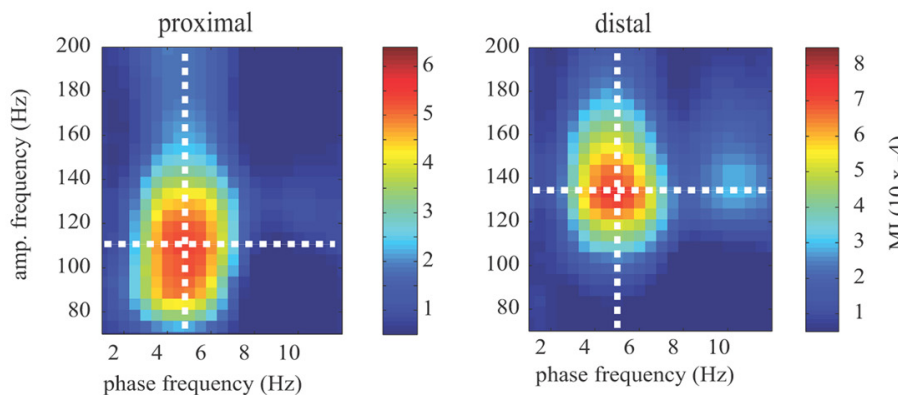

Figure 9. Intrinsic topographical organization of slow and fast gamma in the subiculum. $A$, Diagrammatic representation of the whole hippocampus with arrows indicating the axes within which theta, SG, and FG coherence was measured. S, Septal; T, temporal. $\boldsymbol{B}$, The topography of theta, SG, and FG. Coherence was measured in the proximal-distal (blue), septal-temporal (red), and deep-superficial subicular axes (black). Coherence is plotted as a function of absolute distance. ${ }^{*} p<0.05,{ }^{*} p<0.01$. Note that $S G$ coherence is reduced with distance but is still above the significance threshold (the color above each point indicates the post hoc comparison between the deep-superficial axis and the other color-coded axes). FG coherence drops below the significance level at distances $>300 \mu \mathrm{m}$. The dashed line is the 95\% significance threshold. C, Spike-LFP phase locking between local and distant $(1 \mathrm{~mm})$ regions of the subiculum. $i$, Diagrammatic representation of the experiment in which LFPs from local and distant electrodes were recorded together with spikes next to the local electrode. ii, The phase-locking strength (mean resultant length) is shown and compared between the local LFP electrode and the distant LFP electrode for SG. Filtered LFP traces are shown for SG from the local and distant electrode. All spikes were phase locked to the local SG, and 13 of 21 cells were phase locked to SG in the distant subicular electrode. iii, The same as in ii for FG. Note how the three spikes fire on a similar phase of the local FG but on different phases of FG from the distant electrode because of a slightly slower $\mathrm{FG}$ network frequency. $\boldsymbol{D}$, On the left is a representative histological section showing the location of electrodes located in the proximal and distal subiculum. The mean normalized theta MI plots are shown for the proximal and distal subiculum. The proximal and distal subiculum prefer different ratios of $S G$ and $F G$, and the distal subiculum has a greater preference for $\mathrm{FG}$ whereas the proximal subiculum preferred $\mathrm{SG}$. Black lines are the mean, and gray shading indicates the SE. $\boldsymbol{E}$, An example phase-amplitude modulation index plot is shown for simultaneously recorded field potentials in the proximal and distal subiculum, demonstrating that the distal subiculum exhibits a higher $\mathrm{FG}$ frequency.

subiculum are generated by different synaptic mechanisms, because SG was modulated by excitatory and inhibitory blockade, whereas FG was mediated primarily by inhibition. Surprisingly, gamma rhythms were not consistently present in either the CA3 or CA1 area in the complete hippocampal preparation, suggesting that gamma generation in these areas may necessitate external excitatory input and that the level of internal synaptic excitatory and inhibitory activity within CA1 and CA3 may not be sufficient to generate spontaneous gamma rhythms. Furthermore, the extensive recurrent collaterals running in the deep-superficial axis of the subiculum (Witter and Groenewegen, 1990; Harris and Stewart, 2001; Harris et al., 2001), together with the greater firing rate (Sharp and Green, 1994; Sharp, 1999) and intrinsic capacity for bursting of subicular cells (Stewart and Wong, 1993; Staff et al., 2000), likely provide the unique neuroconnective substrate for the most robust generation of all three rhythms (theta, SG, FG) in the subiculum.

Collectively, the data presented here demonstrate that the subiculum is one of three regions of the hippocampal formation (in addition to CA3 and the MEC) that exhibits prominent gamma generating properties, with two fundamental frequencies matching those in the MEC (FG) and CA3 (SG). Therefore, the subiculum could serve as a comparator (similar to CA1) (Vinogradova, 2001), functioning to inform thalamic, hypothalamic, and other cortical circuits with information regarding the encoding or retrieval state of the hippocampus.

\section{Spontaneous and independent cross- frequency coupling in vitro}

It has been reported previously that the phase-amplitude coupling between theta and gamma rhythms is important for learning and memory (Canolty et al., 2006; Tort et al., 2009; Axmacher et al., 2010). In the CA1 region in vivo, SG and FG arise on separate theta phases and on different theta cycles (Colgin et al., 2009). This independence is thought to reflect the encoding (FG from the MEC) or retrieval (SG from CA3) state of the hippocampus (Colgin et al., 2009; Fries, 2009). The data presented here demonstrate that cross-frequency coupling between theta and gamma can occur in the subiculum. The network requirements for phase-amplitude coupling remain unknown. However, the data shown here suggest that phase-amplitude coupling in the subiculum is an intrinsic phenomenon, whereas in areas CA3 and CA1, phase-amplitude coupling may require additional synaptic inputs (thereby increasing overall network activity) (Gloveli et al., 2005).

SG and FG in the CA1 are independent and reflect different forms of synaptic input arising from CA3 and the MEC, respectively 
(Colgin et al., 2009). SG and FG in the subiculum were only separated by $\sim 10^{\circ}$, which may reflect genuine differences between the subiculum and CA1 in the phase when SG and FG normally arise, or it could be attributable to a lack of strong afferent inputs in this preparation that could induce phase shifts in the theta phase in which SG or FG occurs. Interestingly, separate populations of CA1 neurons phase lock to SG or FG but rarely to both (Colgin et al., 2009). In contrast, single subicular neurons can phase lock to both gamma frequencies, whereas phase locking in one frequency can arise without significant activity in the other gamma band (Fig. 7). This would be important in vivo because CA1 should provide a constant bombardment of subicular circuits with both SG and FG depending on inputs from CA3 and MEC (Colgin et al., 2009). The presence of two intrinsic gamma generators in subiculum could function to match the frequency of CA1 input to most effectively route information out of hippocampal circuits and to inform target brain regions of the intrinsic state of the hippocampus.

\section{Topography of subicular activity}

The subiculum displays a marked topography of its inputs and outputs. Specifically, the proximal subiculum receives input from the distal CA1 and projects to the lateral entorhinal cortex (LEC), whereas the distal subiculum receives input from the proximal CA1 and projects to the MEC (van Groen and Wyss, 1990; Witter et al., 1990; Naber et al., 2001; Kloosterman et al., 2003). The proximal CA1 (which receives the most dense MEC input) is the most spatially tuned region of CA1 and phase locks most robustly to theta in the MEC (Henriksen et al., 2010) and, in accordance, the neurons in the distal subiculum have greater spatial tuning, higher firing rate (Sharp and Green, 1994), and more bursting (Staff et al., 2000). Furthermore, grid cells are found mainly within the MEC (Hafting et al., 2005) and not in the LEC. Therefore, spatial memory appears to involve specific topographical loops between the MEC and hippocampus (CA1), which use FG to route information between areas. We showed that the distal subiculum preferred to phase lock to theta using FG, therefore suggesting that the MEC-proximal CA1-distal subiculum loop may use FG to route spatially related information through this circuit. In contrast, the proximal subiculum-LEC would function most effectively using SG. Therefore, these data demonstrate that different pathways in the cortical hippocampal circuit may use different gamma frequencies to selectively transmit information. In addition, the segregation of information processing between the pathways using the proximal and distal subicular regions would be ensured by different frequencies of FG (Fig. 9).

\section{Conclusions}

Hippocampal theta and gamma rhythms have an important role in the synchronization of cortical and subcortical neural assemblies involved in a diverse set of cognitive functions (Vorel et al., 2001; Seidenbecher et al., 2003; Siapas et al., 2005; DeCoteau et al., 2007; Colgin et al., 2009; Popa et al., 2010). Although the importance of the subiculum in memory, emotion, and stress are well known (Herman and Cullinan, 1997; O'Mara et al., 2009; Ulrich-Lai and Herman, 2009), little is known regarding how network activity in the subiculum could regulate hippocampal outputs. The intrinsic topographical organization of independent SG and FG by subicular networks might therefore function as an interface to inform the remainder of the brain as to the ongoing state of the hippocampus.

\section{References}

Atallah BV, Scanziani M (2009) Instantaneous modulation of gamma oscillation frequency by balancing excitation with inhibition. Neuron 62:566-577. Axmacher N, Henseler MM, Jensen O, Weinreich I, Elger CE, Fell J (2010)
Cross-frequency coupling supports multi-item working memory in the human hippocampus. Proc Natl Acad Sci U S A 107:3228-3233.

Berens P (2009) CircStat: a Matlab toolbox for circular statistics. J Statistical Software 31:1-21.

Bokil H, Andrews P, Kulkarni JE, Mehta S, Mitra PP (2010) Chronux: a platform for analyzing neural signals. J Neurosci Methods 192:146-151.

Bragin A, Jandó G, Nádasdy Z, Hetke J, Wise K, Buzsáki G (1995) Gamma $(40-100 \mathrm{~Hz})$ oscillation in the hippocampus of the behaving rat. J Neurosci 15:47-60.

Brotons-Mas JR, Montejo N, O’Mara SM, Sanchez-Vives MV (2010) Stability of subicular place fields across multiple light and dark transitions. Eur J Neurosci 32:648-658.

Canolty RT, Edwards E, Dalal SS, Soltani M, Nagarajan SS, Kirsch HE, Berger MS, Barbaro NM, Knight RT (2006) High gamma power is phaselocked to theta oscillations in human neocortex. Science 313:1626-1628.

Cappaert NL, Wadman WJ, Witter MP (2007) Spatiotemporal analyses of interactions between entorhinal and CA1 projections to the subiculum in rat brain slices. Hippocampus 17:909-921.

Chrobak JJ, Buzsáki G (1998) Gamma oscillations in the entorhinal cortex of the freely behaving rat. J Neurosci 18:388-398.

Colgin LL, Denninger T, Fyhn M, Hafting T, Bonnevie T, Jensen O, Moser MB, Moser EI (2009) Frequency of gamma oscillations routes flow of information in the hippocampus. Nature 462:353-357.

Colling SB, Stanford IM, Traub RD, Jefferys JG (1998) Limbic gamma rhythms. I. Phase-locked oscillations in hippocampal CA1 and subiculum. J Neurophysiol 80:155-161.

Csicsvari J, Jamieson B, Wise KD, Buzsáki G (2003) Mechanisms of gamma oscillations in the hippocampus of the behaving rat. Neuron 37:311-322.

Cunningham MO, Davies CH, Buhl EH, Kopell N, Whittington MA (2003) Gamma oscillations induced by kainate receptor activation in the entorhinal cortex in vitro. J Neurosci 23:9761-9769.

Cunningham MO, Halliday DM, Davies CH, Traub RD, Buhl EH, Whittington MA (2004) Coexistence of gamma and high-frequency oscillations in rat medial entorhinal cortex in vitro. J Physiol 559:347-353.

Deadwyler SA, Hampson RE (2004) Differential but complementary mnemonic functions of the hippocampus and subiculum. Neuron 42:465-476.

DeCoteau WE, Thorn C, Gibson DJ, Courtemanche R, Mitra P, Kubota Y, Graybiel AM (2007) Learning-related coordination of striatal and hippocampal theta rhythms during acquisition of a procedural maze task. Proc Natl Acad Sci U S A 104:5644-5649.

Fisahn A, Pike FG, Buhl EH, Paulsen O (1998) Cholinergic induction of network oscillations at $40 \mathrm{~Hz}$ in the hippocampus in vitro. Nature 394:186-189.

Fries P (2009) The model- and the data-gamma. Neuron 64:601-602.

Funahashi M, Stewart M (1998) Properties of gamma-frequency oscillations initiated by propagating population bursts in retrohippocampal regions of rat brain slices. J Physiol 510:191-208.

Gloveli T, Dugladze T, Rotstein HG, Traub RD, Monyer H, Heinemann U, Whittington MA, Kopell NJ (2005) Orthogonal arrangement of rhythmgenerating microcircuits in the hippocampus. Proc Natl Acad Sci U S A 102:13295-13300.

Goutagny R, Jackson J, Williams S (2009) Self-generated theta oscillations in the hippocampus. Nat Neurosci 12:1491-1493.

Hafting T, Fyhn M, Molden S, Moser MB, Moser EI (2005) Microstructure of a spatial map in the entorhinal cortex. Nature 436:801-806.

Harris E, Stewart M (2001) Intrinsic connectivity of the rat subiculum. II. Properties of synchronous spontaneous activity and a demonstration of multiple generator regions. J Comp Neurol 435:506-518.

Harris E, Witter MP, Weinstein G, Stewart M (2001) Intrinsic connectivity of the rat subiculum. I. Dendritic morphology and patterns of axonal arborization by pyramidal neurons. J Comp Neurol 435:490-505.

Henriksen EJ, Colgin LL, Barnes CA, Witter MP, Moser MB, Moser EI (2010) Spatial representation along the proximodistal axis of CA1. Neuron 68:127-137.

Herman JP, Cullinan WE (1997) Neurocircuitry of stress: central control of the hypothalamo-pituitary-adrenocortical axis. Trends Neurosci 20:78-84.

Jacobs J, Kahana MJ (2009) Neural representations of individual stimuli in humans revealed by gamma-band electrocorticographic activity. J Neurosci 29:10203-10214.

Jacobs J, Kahana MJ, Ekstrom AD, Fried I (2007) Brain oscillations control timing of single-neuron activity in humans. J Neurosci 27:3839-3844.

Jutras MJ, Fries P, Buffalo EA (2009) Gamma-band synchronization in the macaque hippocampus and memory formation. J Neurosci 29:12521-12531. 
Khalilov I, Esclapez M, Medina I, Aggoun D, Lamsa K, Leinekugel X, Khazipov R, Ben-Ari Y (1997) A novel in vitro preparation: the intact hippocampal formation. Neuron 19:743-749.

Khalilov I, Holmes GL, Ben-Ari Y (2003) In vitro formation of a secondary epileptogenic mirror focus by interhippocampal propagation of seizures. Nat Neurosci 6:1079-1085.

Kloosterman F, Witter MP, Van Haeften T (2003) Topographical and laminar organization of subicular projections to the parahippocampal region of the rat. J Comp Neurol 455:156-171.

Kramer MA, Tort AB, Kopell NJ (2008) Sharp edge artifacts and spurious coupling in EEG frequency comodulation measures. J Neurosci Methods 170:352-357.

Lisman JE, Idiart MA (1995) Storage of $7 \pm 2$ short-term memories in oscillatory subcycles. Science 267:1512-1515.

Manseau F, Goutagny R, Danik M, Williams S (2008) The hippocamposeptal pathway generates rhythmic firing of GABAergic neurons in the medial septum and diagonal bands: an investigation using a complete septohippocampal preparation in vitro. J Neurosci 28:4096-4107.

Masimore B, Kakalios J, Redish AD (2004) Measuring fundamental frequencies in local field potentials. J Neurosci Methods 138:97-105.

Menendez de la Prida L (2003) Control of bursting by local inhibition in the rat subiculum in vitro. J Physiol 549:219-230.

Montgomery SM, Buzsáki G (2007) Gamma oscillations dynamically couple hippocampal CA3 and CA1 regions during memory task performance. Proc Natl Acad Sci U S A 104:14495-14500.

Montgomery SM, Sirota A, Buzsáki G (2008) Theta and gamma coordination of hippocampal networks during waking and rapid eye movement sleep. J Neurosci 28:6731-6741.

Naber PA, Witter MP (1998) Subicular efferents are organized mostly as parallel projections: a double-labeling, retrograde-tracing study in the rat. J Comp Neurol 393:284-297.

Naber PA, Lopes da Silva FH, Witter MP (2001) Reciprocal connections between the entorhinal cortex and hippocampal fields $\mathrm{CA} 1$ and the subiculum are in register with the projections from CA1 to the subiculum. Hippocampus 11:99-104.

O’Mara SM, Sanchez-Vives MV, Brotons-Mas JR, O’Hare E (2009) Roles for the subiculum in spatial information processing, memory, motivation and the temporal control of behaviour. Prog Neuropsychopharmacol Biol Psychiatry 33:782-790.

Oren I, Mann EO, Paulsen O, Hájos N (2006) Synaptic currents in anatomically identified CA3 neurons during hippocampal gamma oscillations in vitro. J Neurosci 26:9923-9934.

Oren I, Hájos N, Paulsen O (2010) Identification of the current generator underlying cholinergically induced gamma frequency field potential oscillations in the hippocampal CA3 region. J Physiol 588:785-797.

Popa D, Duvarci S, Popescu AT, Léna C, Paré D (2010) Coherent amygdalocortical theta promotes fear memory consolidation during paradoxical sleep. Proc Natl Acad Sci U S A 107:6516-6519.

Quilichini P, Sirota A, Buzsáki G (2010) Intrinsic circuit organization and theta-gamma oscillation dynamics in the entorhinal cortex of the rat. J Neurosci 30:11128-11142.

Risold PY, Swanson LW (1996) Structural evidence for functional domains in the rat hippocampus. Science 272:1484-1486.

Rutishauser U, Ross IB, Mamelak AN, Schuman EM (2010) Human memory strength is predicted by theta-frequency phase-locking of single neurons. Nature 464:903-907.

Sauseng P, Klimesch W, Heise KF, Gruber WR, Holz E, Karim AA, Glennon M, Gerloff C, Birbaumer N, Hummel FC (2009) Brain oscillatory substrates of visual short-term memory capacity. Curr Biol 19:1846-1852.

Sederberg PB, Schulze-Bonhage A, Madsen JR, Bromfield EB, McCarthy DC, Brandt A, Tully MS, Kahana MJ (2007) Hippocampal and neocortical gamma oscillations predict memory formation in humans. Cereb Cortex 17:1190-1196.

Seidenbecher T, Laxmi TR, Stork O, Pape HC (2003) Amygdalar and hippocampal theta rhythm synchronization during fear memory retrieval. Science 301:846-850.

Senior TJ, Huxter JR, Allen K, O’Neill J, Csicsvari J (2008) Gamma oscillatory firing reveals distinct populations of pyramidal cells in the CA1 region of the hippocampus. J Neurosci 28:2274-2286.
Sharp PE (1999) Comparison of the timing of hippocampal and subicular spatial signals: implications for path integration. Hippocampus 9:158-172.

Sharp PE, Green C (1994) Spatial correlates of firing patterns of single cells in the subiculum of the freely moving rat. J Neurosci 14:2339-2356.

Siapas AG, Lubenov EV, Wilson MA (2005) Prefrontal phase locking to hippocampal theta oscillations. Neuron 46:141-151.

Sirota A, Montgomery S, Fujisawa S, Isomura Y, Zugaro M, Buzsáki G (2008) Entrainment of neocortical neurons and gamma oscillations by the hippocampal theta rhythm. Neuron 60:683-697.

Staff NP, Jung HY, Thiagarajan T, Yao M, Spruston N (2000) Resting and active properties of pyramidal neurons in subiculum and CA1 of rat hippocampus. J Neurophysiol 84:2398-2408.

Stanford IM, Traub RD, Jefferys JG (1998) Limbic gamma rhythms. II. Synaptic and intrinsic mechanisms underlying spike doublets in oscillating subicular neurons. J Neurophysiol 80:162-171.

Stewart M, Wong RK (1993) Intrinsic properties and evoked responses of guinea pig subicular neurons in vitro. J Neurophysiol 70:232-245.

Sullivan D, Csicsvari J, Mizuseki K, Montgomery S, Diba K, Buzsáki G (2011) Relationships between hippocampal sharp waves, ripples, and fast gamma oscillation: influence of dentate and entorhinal cortical activity. J Neurosci 31:8605-8616.

Tamamaki N, Nojyo Y (1990) Disposition of the slab-like modules formed by axon branches originating from single CA1 pyramidal neurons in the rat hippocampus. J Comp Neurol 291:509-519.

Tiesinga P, Sejnowski TJ (2009) Cortical enlightenment: are attentional gamma oscillations driven by ING or PING? Neuron 63:727-732.

Tort AB, Kramer MA, Thorn C, Gibson DJ, Kubota Y, Graybiel AM, Kopell NJ (2008) Dynamic cross-frequency couplings of local field potential oscillations in rat striatum and hippocampus during performance of a T-maze task. Proc Natl Acad Sci U S A 105:20517-20522.

Tort AB, Komorowski RW, Manns JR, Kopell NJ, Eichenbaum H (2009) Theta-gamma coupling increases during the learning of item-context associations. Proc Natl Acad Sci U S A 106:20942-20947.

Traub RD, Bibbig A, LeBeau FE, Buhl EH, Whittington MA (2004) Cellular mechanisms of neuronal population oscillations in the hippocampus in vitro. Annu Rev Neurosci 27:247-278.

Tukker JJ, Fuentealba P, Hartwich K, Somogyi P, Klausberger T (2007) Cell type-specific tuning of hippocampal interneuron firing during gamma oscillations in vivo. J Neurosci 27:8184-8189.

Ulrich-Lai YM, Herman JP (2009) Neural regulation of endocrine and autonomic stress responses. Nat Rev Neurosci 10:397-409.

van Groen T, Wyss JM (1990) The postsubicular cortex in the rat: characterization of the fourth region of the subicular cortex and its connections. Brain Res 529:165-177.

van Strien NM, Cappaert NL, Witter MP (2009) The anatomy of memory: an interactive overview of the parahippocampal-hippocampal network. Nat Rev Neurosci 10:272-282.

Vinogradova OS (2001) Hippocampus as comparator: role of the two input and two output systems of the hippocampus in selection and registration of information. Hippocampus 11:578-598.

Vorel SR, Liu X, Hayes RJ, Spector JA, Gardner EL (2001) Relapse to cocaine-seeking after hippocampal theta burst stimulation. Science 292:1175-1178.

Whittington MA, Traub RD, Jefferys JG (1995) Synchronized oscillations in interneuron networks driven by metabotropic glutamate receptor activation. Nature 373:612-615.

Witter MP (2006) Connections of the subiculum of the rat: topography in relation to columnar and laminar organization. Behav Brain Res 174:251-264.

Witter MP, Groenewegen HJ (1990) The subiculum: cytoarchitectonically a simple structure, but hodologically complex. Prog Brain Res 83:47-58.

Witter MP, Ostendorf RH, Groenewegen HJ (1990) Heterogeneity in the dorsal subiculum of the rat. Distinct neuronal zones project to different cortical and subcortical targets. Eur J Neurosci 2:718-725.

Wu CP, Huang HL, Asl MN, He JW, Gillis J, Skinner FK, Zhang L (2006) Spontaneous rhythmic field potentials of isolated mouse hippocampalsubicular-entorhinal cortices in vitro. J Physiol 576:457-476.

Wyart V, Tallon-Baudry C (2008) Neural dissociation between visual awareness and spatial attention. J Neurosci 28:2667-2679. 